\title{
A DETAILED COMPARISON BETWEEN THE OBSERVED AND SYNTHESIZED PROPERTIES OF A SIMULATED TYPE II SPICULE
}

\author{
Juan Martínez-Sykora ${ }^{1,2,3}$, Bart De Pontieu ${ }^{1}$, Jorrit Leenaarts ${ }^{2}$, Tiago M. D. Pereira ${ }^{1,4}$, Mats $_{\text {Carlsson }}^{2}$, \\ Viggo Hansteen $^{2}$, Julie V. Stern ${ }^{1}$, Hui Tian ${ }^{5}$, Scott W. McIntosh ${ }^{6}$, and Luc Rouppe Van Der Voort ${ }^{2}$ \\ ${ }^{1}$ Lockheed Martin Solar and Astrophysics Laboratory, Palo Alto, CA 94304, USA; j.m.sykora@ astro.uio.no \\ 2 Institute of Theoretical Astrophysics, University of Oslo, P.O. Box 1029 Blindern, N-0315 Oslo, Norway \\ ${ }^{3}$ Bay Area Environmental Research Institute, Sonoma, CA 95476, USA \\ ${ }^{4}$ NASA Ames Research Center, Moffett Field, CA 94035, USA \\ ${ }^{5}$ Harvard-Smithsonian Center for Astrophysics, Cambridge, MA 02138, USA \\ ${ }^{6}$ High Altitude Observatory, National Center for Atmospheric Research, Boulder, CO 80307, USA \\ Received 2013 January 16; accepted 2013 May 7; published 2013 June 17
}

\begin{abstract}
We have performed a three-dimensional radiative MHD simulation of the solar atmosphere. This simulation shows a jet-like feature that shows similarities to the type II spicules observed for the first time with Hinode's Solar Optical Telescope. Rapid blueshifted events (RBEs) on the solar disk are associated with these spicules. Observational results suggest they may contribute significantly in supplying the corona with hot plasma. We perform a detailed comparison of the properties of the simulated jet with those of type II spicules (observed with Hinode) and RBEs (with ground-based instruments). We analyze a wide variety of synthetic emission and absorption lines from the simulations including chromospheric (Ca II $8542 \AA, \mathrm{Ca}$ II $\mathrm{H}$, and $\mathrm{H} \alpha$ ) to transition region and coronal temperatures $(10,000 \mathrm{~K}$ to several million K). We compare their synthetic intensities, line profiles, Doppler shifts, line widths, and asymmetries with observations from Hinode/SOT and EIS, SOHO/SUMER, the Swedish $1 \mathrm{~m}$ Solar Telescope, and SDO/AIA. Many properties of the synthetic observables resemble the observations, and we describe in detail the physical processes that lead to these observables. Detailed analysis of the synthetic observables provides insight into how observations should be analyzed to derive information about physical variables in such a dynamic event. For example, we find that line-of-sight superposition in the optically thin atmosphere requires the combination of Doppler shifts and spectral line asymmetry to determine the velocity in the jet. In our simulated type II spicule, the lifetime of the asymmetry of the transition region lines is shorter than that of the coronal lines. Other properties differ from the observations, especially in the chromospheric lines. The mass density of the part of the spicule with a chromospheric temperature is too low to produce significant opacity in chromospheric lines. The synthetic Ca II $8542 \AA$ and $\mathrm{H} \alpha$ profiles therefore do not show signal resembling RBEs. These and other discrepancies are described in detail, and we discuss which mechanisms and physical processes may need to be included in the MHD simulations to mimic the thermodynamic processes of the chromosphere and corona, in particular to reproduce type II spicules.
\end{abstract}

Key words: magnetohydrodynamics (MHD) - radiative transfer - Sun: atmosphere - Sun: chromosphere - Sun: corona - Sun: transition region

Online-only material: color figures

\section{INTRODUCTION}

The $\mathrm{H} \alpha$ line, which reveals the relatively cool plasma in the chromosphere, shows that the upper chromosphere is dominated by highly dynamic spicules. Our view of spicules has been revolutionized thanks to advanced instruments, such as the Hinode satellite (Kosugi et al. 2007) and the Swedish $1 \mathrm{~m}$ Solar Telescope (SST), where adaptive optics and image postprocessing are necessary (van Noort et al. 2005). Using Hinode observations, De Pontieu et al. (2007b) distinguished at least two different types of spicules.

The first type of spicules (so-called type I) reaches heights above the photosphere of 2-9 Mm, has a lifetime of 3-10 minutes, and shows up- and downward motion (Beckers 1968; Suematsu et al. 1995). Type I spicules are probably the counterpart of the dynamic fibrils on the disk and they follow a parabolic path in space and time. This evolution is caused by a magneto-acoustic shock wave passing through the chromosphere (Shibata et al. 1982; Shibata \& Suematsu 1982; De Pontieu et al. 2004; Hansteen et al. 2006; De Pontieu et al. 2007a; Heggland et al. 2007; Martínez-Sykora et al. 2009; Matsumoto \& Shibata 2010, among others). Although the formation of type I spicules seems to be well understood, even the most recent studies do not include various processes that may play an important role in chromospheric dynamics and/or diagnostics, such as time dependent ionization, generalized Ohm's law, three-dimensional (3D) radiative transfer with scattering, etc. The impact of these various effects on type I spicule evolution should be tested and compared with observations.

The second type of spicules (so-called type II) reaches greater heights $(\sim 6.5 \mathrm{Mm})$ and has shorter lifetimes $(\sim 100 \mathrm{~s})$ than type I spicules (De Pontieu et al. 2007b; Pereira et al. 2012). In addition, type II spicules show apparent upward motions of order $50-100 \mathrm{~km} \mathrm{~s}^{-1}$ and at the end of their life they usually suffer a rapid fading in images taken in chromospheric lines. The counterparts on the disk appear as rapidly moving absorbing features in the blue wing of chromospheric lines (Langangen et al. 2008; Rouppe van der Voort et al. 2009). In contrast to type I spicules, type II spicules are not well understood. For example, some observations suggest the type II spicules are impulsively and continuously accelerated while being heated to at least transition region temperatures (De Pontieu et al. 2009, 2011). Recent observations (Sekse et al. 2012) add complexity by indicating that some type II spicules also show a decrease or a 
more complex velocity dependence with height. These spicules seem to show emission in transition region and coronal lines (De Pontieu et al. 2011); but Madjarska et al. (2011), using lower signal-to-noise observations, suggest that this emission comes from cold plasma. In addition, SDO/AIA observations show that some type II spicules observed at the limb in the channel $304 \AA$ (He II $304 \AA$ ) but it is unclear how common this is. The lack of detailed observational constraints poses significant challenges to theoretical modeling. As a result there is currently no model that can describe all aspects of type II spicule formation.

Other motions, apart from the apparent upward motion, are observed in type II spicules. For instance, these features show swaying motions at the limb of order $10-30 \mathrm{~km} \mathrm{~s}^{-1}$, suggesting Alfvénic waves as observed in the chromosphere by De Pontieu et al. (2007c) and Okamoto \& De Pontieu (2011), and in the transition region and corona by McIntosh et al. (2011). Suematsu et al. (2008) suggested that some spicules show multi-thread structure as result of a possible rotation. The torsional motions along spicules were suggested in earlier reports (Beckers 1972), but only recently unequivocally established using high resolution spectroscopy at the limb by De Pontieu et al. (2012). The latter establish that type II spicules show torsional motions of order $25-30 \mathrm{~km} \mathrm{~s}^{-1}$. Similar torsional motions were observed in transition region lines in macrospicules and explosive events using both Dopplergrams and line profiles (Curdt \& Tian 2011).

As a result of the highly dynamic and finely structured nature of this type of spicules and the complex physical processes governing the chromosphere (radiation, time-dependent ionization, partial ionization effects, etc.), our understanding of type II spicules remains limited, as mentioned above. It is important that a successful model of these jets also explains the impact of type II spicules on the various atmospheric layers (from the chromosphere to the lower corona). De Pontieu et al. (2007b) and Sterling et al. (2010) suggested that type II spicules are driven by magnetic reconnection. Another possible candidate, also more elaborate, was presented by Martínez-Sykora et al. (2011), where the chromospheric plasma is ejected as a result of being squeezed by the magnetic tension resulting from flux emergence. The resulting jet shows evidence of both high velocities in the chromosphere and heating of plasma to coronal temperatures. On this basis, the jet was tentatively identified as a type II spicule. However, a full and detailed comparison of the properties of various synthetic observables of the jet with observations of spicules and their counterparts has not yet been performed.

In this paper, we expand on the work done by Martínez-Sykora et al. (2011) by comparing synthetic images and spectra of the simulated type II spicule with observations from Hinode, Solar Dynamics Observatory (SDO; Lemen et al. 2012), SST, and Solar and Heliospheric Observatory (SOHO). The description of the code and the setup of the simulation used to simulate the candidate type II spicule are detailed in Section 2. In order to compare with observations, we calculated synthetic observables as described in Section 2.2. The various observations and the data reduction are described in Section 3. Section 4.1 details the synthetic chromospheric spectra and images in Ca II $8542 \AA$ and $\mathrm{H} \alpha$ and the comparison with observations. The synthetic transition region and coronal observables of the spicule are studied in Section 4.2; they are compared with observations in Section 4.3. We finish with a discussion in Section 5.

\section{NUMERICAL METHOD AND SETUP}

The MHD equations are solved in a 3D computational domain using the Oslo Stagger Code, which is the predecessor of the Bifrost code (Gudiksen et al. 2011). The core of the numerical methods is the same and has been described also in detail by Dorch \& Nordlund (1998), Mackay \& Galsgaard (2001), Martínez-Sykora et al. (2008), and Martínez-Sykora et al. (2009). Essentially the code solves the partial spatial derivatives using a sixth order accurate method. In addition, a fifth order interpolation scheme is used if variables are needed at other locations than their defined position with respect to the grid. Time stepping of the MHD equations is performed using a modified version of the third order predictor-corrector procedure detailed by Hyman et al. (1979). Numerical noise is suppressed by adding a high-order artificial magnetic hyper-diffusion and -viscosity. The magnetic and viscous energy dissipated through the hyper-diffusive operator is self-consistently implemented into the energy conservative equation.

From the photosphere to the lower chromosphere the radiative flux divergence is calculated by wavelength and angle integration. The opacities and emissivities are assumed to be isotropic. In order to solve the radiative transfer equation, we assume four group mean local thermodynamic equilibrium (LTE) opacities to cover the entire spectrum (Nordlund 1982). The transfer equation is reformulated calculating a mean source function in each bin. In addition, an approximate coherent scattering and a contribution from the thermal emissivity are included in the source function. The 3D scattering problem is solved following (Skartlien 2000), i.e., it is solved by iteration using one-ray in the integral for the mean intensity.

For the middle and upper chromosphere, non-LTE radiative transfer losses from hydrogen continua, hydrogen lines, and lines from singly ionized calcium are calculated using (Carlsson \& Leenaarts 2012) recipes based on a one-dimensional (1D) dynamical chromospheric model (Carlsson \& Stein 1992, 1994, 1997, 2002).

Optically thin radiative losses are calculated in the upper chromosphere, transition region, and corona assuming the coronal approximation: hydrogen, helium, carbon oxygen, neon, and iron elements are included. Atomic data is obtained from the HAO spectral diagnostics package (Judge \& Meisner 1994).

The thermal conduction along the magnetic field lines is treated by operator splitting. The conductive operator is solved using the implicit Crank-Nicolson method and is iterated to convergence using a multi-grid solver (Gudiksen et al. 2011).

\subsection{Initial and Boundary Conditions}

The simulation discussed in this paper was used to study flux emergence in Martínez-Sykora et al. (2008, 2009), type I spicules in Martínez-Sykora et al. (2009) and the driving mechanism of a jet that resembles a type II spicule in Martínez-Sykora et al. (2011). Here we calculate synthetic observables of this jet and describe the results. This simulation was named as "B1" in the previous work. The domain spans from the upper convection zone (1.5 Mm below the photosphere) to the lower corona (14 Mm above the photosphere). The horizontal extent is $16 \mathrm{Mm}$ in the chosen $x$-direction and $8 \mathrm{Mm}$ in the $y$-direction, as shown in Figure 1. This figure shows a selection of magnetic field lines (red), velocity streamlines (blue), and the emissivity of He II $304 \AA$ (green-red semi-transparent color scheme) and Fe XIV $211 \AA$ (gray-isosurface) at $t=1590 \mathrm{~s}$. The domain is 

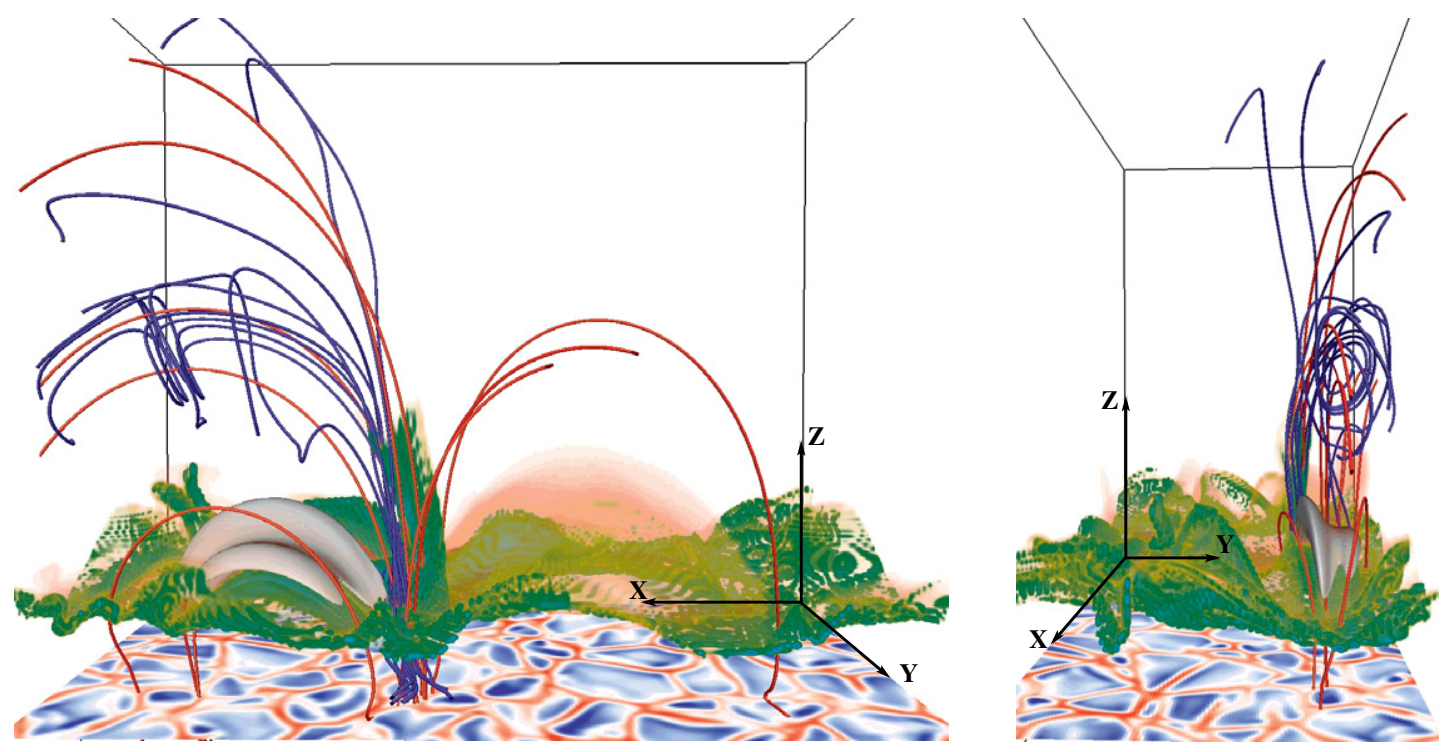

Figure 1. Characterization of our radiation-MHD simulation. The panels show two 3D visualization of the computational domain at $t=1590 \mathrm{~s}$. The vertical velocity in the photosphere is shown with a red-blue color scheme $\left([-2,2] \mathrm{km} \mathrm{s}^{-1}\right.$; blue are upflowing granules). Selected magnetic field lines are shown in red, selected velocity streamlines are blue. An isosurface of Fe XIV $211 \AA ̊$ emission is shown in gray. The He II $304 \AA$ emissivity is shown with the red-green semi-transparent color scheme with green corresponding to high emissivity.

(A color version of this figure is available in the online journal.)

evaluated in $256 \times 128 \times 160$ grid points using a uniform grid in the horizontal direction (i.e., the horizontal grid spacing is $65 \mathrm{~km}$ ) and a non-uniform grid in the vertical direction, where the spacing becomes larger at coronal heights as gradients are smaller and the scale heights larger than lower in the atmosphere (i.e., the minimal vertical grid spacing is $32 \mathrm{~km}$ ). After the transients from the initialization are relaxed, the simulation was run for one hour of solar time.

The initial mean magnetic intensity in the photosphere is $\sim 160 \mathrm{G}$. The magnetic field is distributed in loop-shapes in the corona where their footpoints are rooted in two bands along the $y$-axis in the photosphere $(x \approx 7 \mathrm{Mm}$ and $x \approx 13 \mathrm{Mm})$. As a result of this the magnetic field lines in the corona are roughly oriented along the $x$-axis (see Figure 1). This figure corresponds to the instant $t=1590 \mathrm{~s}$, which shows the ejected type II spicule as a vertical green finger in the left-hand panel.

The trigger of the series of events that lead to the type II spicule is a non-twisted flux tube that we introduced into the computational domain through its lower boundary, as detailed in Section 3.2 by Martínez-Sykora et al. (2008). This horizontal flux tube is injected in a band along the $y$-axis with a diameter of $1.5 \mathrm{Mm}$ at $x=8 \mathrm{Mm}$. Note that the emerging field is mostly perpendicular to the orientation of the initial coronal loops shown in Figure 1. The input parameters of the flux emergence are shown in Martínez-Sykora et al. (2009). The detailed description of how the flux emergence drives the type II spicule is in Martínez-Sykora et al. (2011).

\subsection{Methods: Synthetic Diagnostics}

To analyze the emergent emission of the simulated atmosphere, we calculate synthetic images and profiles of the chromospheric Ca II $8542 \AA$, Ca II H $3968 \AA$, and $\mathrm{H} \alpha$ lines, which have been used to study rapid blueshifted events (RBEs) and type II spicules with Hinode (Ca II H $3968 \AA$ ) and SST (Rouppe van der Voort et al. 2009; Sekse et al. 2012). In addition, we synthesized the emission in different EUV lines that form at transition region and coronal temperatures, including the dominant lines of the various $S D O /$ AIA channels and various Hinode/EIS and $\mathrm{SOHO} / \mathrm{SUMER}$ lines.

The Ca II $8542 \AA$ and Ca II H diagnostics have been calculated using the $R H$ code (Uitenbroek 2001). An MPI-parallelized version of this code was used to solve the non-LTE radiative transfer problem in $1 \mathrm{D}$ on a column-by-column basis $(1.5 \mathrm{D}$ approximation), for each snapshot of the simulation. Leenaarts \& Carlsson (2009) find that a 1D treatment is a good approximation for $\mathrm{Ca}$ II $\mathrm{H}$ except at the very line core. Thus, its use is justified for wavelength-integrated Ca II filtergrams. A five-level plus continuum model Ca II atom was employed. Using the non-LTE opacity, source function, and radiation field, line profiles were calculated for the top and side view. For these calculations complete redistribution (CRD) was assumed.

We computed the $\mathrm{H} \alpha$ line intensity with the Multi3d code (Leenaarts \& Carlsson 2009) in the same manner as done by Leenaarts et al. (2012). We employed a five-level plus continuum hydrogen model atom and assumed statistical equilibrium. All lines were treated assuming CRD. To mimic the effect of partial redistribution, the $\operatorname{Ly} \alpha$ and $\operatorname{Ly} \beta$ lines absorption profiles are assumed to be Gaussians with Doppler broadening only. The radiation field was computed in full $3 \mathrm{D}$, which is essential for the $\mathrm{H} \alpha$ radiative transfer, a 1.5D approach fails to reveal any chromospheric structure due to lack of lateral smoothing of the radiation field (Leenaarts et al. 2012). Possibly important effects of non-equilibrium hydrogen ionization (Leenaarts et al. 2007; Leenaarts et al. 2012) were not taken into account in the numerical simulations so we used LTE electron densities and temperatures.

Limb observations are synthesized assuming filter profiles from Hinode/SOT (Ca II H 3 A FWHM).

The emission for coronal EUV lines is calculated assuming the optically thin approximation under ionization equilibrium conditions. Hence, the synthetic frequency-integrated intensity in a spectral line is

$$
I(v, w)=\int_{l} A_{b} n_{\mathrm{e}}(v, w, l) n_{H}(v, w, l) G\left(T, n_{\mathrm{e}}\right) d l,
$$


where $l, v$, and $w$ are length along the line-of-sight (LOS), and the position in the surface perpendicular to the LOS, respectively. The synthetic images result by integrating $(l)$, e.g., along the $z$-axis, therefore $v$ and $w$ are in the $x y$ plane. Here $A_{b}, n_{\mathrm{e}}, n_{H}$, and $G\left(T, n_{\mathrm{e}}\right)$ represent the abundance of the emitting element, the electron and the hydrogen densities, and the contribution function, respectively. The electron density is taken from the equation of state lookup table of the simulation. We create a lookup table of the contribution function $\left(G\left(T, n_{\mathrm{e}}\right)\right)$ using the Solarsoft package for IDL ch_synthetic.pro, where the keyword GOFT is selected. Knowing the temperature $(T)$, and the electron density $\left(n_{\mathrm{e}}\right)$ for each grid-point, $G\left(T, n_{\mathrm{e}}\right)$ is obtained by linear interpolation of the lookup table (in log space). To synthesize the plasma emission we use CHIANTI v.7.0 (Dere et al. 2009; Dere 2011) with the ionization balance chianti . ioneq, available in the CHIANTI distribution. We synthesized observations for photospheric abundances (Grevesse \& Sauval 1998).

The synthetic EUV spectral line profiles are computed (as first done for a 3D simulation by Peter et al. 2006) following the methods described in Hansteen et al. (2010), which assume that the lines are optically thin and in ionization equilibrium, as mentioned above.

Once we have emergent spectral line profiles, we use similar techniques as those used by observers to examine our results. We perform a single Gaussian fit to the profile to determine the Doppler shift and the line width. For the asymmetries of the line profiles, we use the red-blue asymmetry, hereafter RB asymmetry (De Pontieu et al. 2009). Briefly, we fit a single Gaussian to the core of the line profile, and determine the line center of the Gaussian fit. The RB asymmetry is calculated by subtracting the integrated intensity within two spectral windows that are identical in size and located at identical wavelength offsets from the line center, with one window toward the red and one toward the blue (Martínez-Sykora et al. 2011a).

Several studies have been done and prove the importance of the time dependent ionization for several of the EUV lines that we are using here (Joselyn \& Holzer 1975; Mariska et al. 1982; Hansteen 1993; Bradshaw 2009; Judge et al. 2012; Olluri et al. 2013). We postpone a detailed study of the (E)UV emission in type II spicules taking into account time dependent ionization to a follow-up paper (see Olluri et al. 2013, for an analysis of time dependence ionization in Bifrost simulations using a similar domain). Some of the results based on EUV lines presented here may thus be subject to the limitation of considering ionization equilibrium conditions.

\section{OBSERVATIONS}

We perform a detailed comparison between these synthetic observables and solar observations. We use observations from the SST (Scharmer et al. 2003) on La Palma using the CRisp Imaging SpectroPolarimeter (Scharmer et al. 2008) instrument. We used $\mathrm{H} \alpha$ and Ca II $8542 \AA$ spectral line profiles obtained on 2012 July 2 at 08:36-09:35 UT. The field of view was $53^{\prime \prime} \times 53^{\prime \prime}$ with a spatial scale of $0^{\prime \prime} 0592$ pixel $^{-1}$. The target was a network region close to disk center at solar coordinates $(x, y) \sim\left(-15^{\prime \prime}, 50^{\prime \prime}\right)$ and $\mu=1$. The CaII $8542 \AA$ line was sampled at 47 spectral positions from $-2717 \mathrm{~m} \AA$ to $+2717 \mathrm{~m} \AA$ with a sampling of $110 \mathrm{~m} \AA$ in the line core region (sparser in the wings). The $\mathrm{H} \alpha$ line profile was sampled at 35 spectral positions in the blue wing from $-2064 \mathrm{~m} \AA$ to $1290 \mathrm{~m} \AA$ with steps of $86 \mathrm{~m} \AA$ in the line core region (sparser in the wings). High spatial resolution was achieved with aid from adaptive optics and image restoration (van Noort et al. 2005).
To study the impact of the type II spicules in the transition region and corona, we used the same data set as in De Pontieu et al. (2011; see this reference for details). In short, the Hinode/ SOT H $\alpha(-868 \mathrm{m \AA})$ images were taken on 2010 April 25 from 01:55:38 to 02:55:02 UTC with a cadence of $12 \mathrm{~s}$ and a pixel size of 0.159 . The data were corrected for dark current, flat-fielded, and co-aligned as a time series. The AIA/SDO images were taken at a cadence of $8 \mathrm{~s}$, with pixels of 0.6 , and exposure times twice as long as during normal AIA operations. To maximize signal to noise we made a new time series at a cadence of $16 \mathrm{~s}$ where each image is the sum of two original images.

Finally, for Doppler shifts and RB asymmetries of the transition region and coronal lines we used a combination of SOHO/SUMER and Hinode/EIS observations. We used a sitand-stare sequence of a quiet Sun region at disk center obtained with SOHO/SUMER on 1996 April 25. These observations show a one hour long sequence of N v $1238 \AA$ spectra that cover a quiet Sun region, including a network region at $y=-30^{\prime \prime}$ to $y=-20^{\prime \prime}$. We use the same data set as Tian et al. (2012) for the Hinode/EIS observations (see the references for details of the data set, calibration, and the methods to calculate the Doppler shifts and RB asymmetries). Unfortunately, current observations do not include simultaneous coverage of clean spectral lines that are formed over a temperature range from the low transition region to the corona.

\section{RESULTS}

Martínez-Sykora et al. (2011) described a physical process of a jet, which in several aspects resembles a type II spicule as described in the current paper. While some of the properties of this jet resemble type II spicules, we only have a single example in our simulation. We discuss possible reasons below. In summary, this process follows naturally as a consequence of the constantly evolving magnetic and thermal environment of the modeled plasma. The spicule is composed of chromospheric material that is rapidly ejected from the chromosphere into the corona, while being heated. The source of the ejection of the chromospheric material is located in a region with large field gradients and intense currents leading to strong Lorentz forces that squeeze the plasma. This increases the pressure gradient leading to strong upflows along the magnetic field lines. Most of the heating comes from magnetic energy dissipation, which appears as a result of the interaction between the ambient field and the emerging fields that straightened as they expanded into the atmosphere. The resulting interaction between the emerging and ambient field lines leads to a tangential discontinuity and subsequently strong heating. This paper focuses on type II spicules, for brevity referred to as "spicules." When we refer to type I spicules, it will be explicit.

\subsection{Synthetic versus Observed Chromospheric Observables}

Martínez-Sykora et al. (2011) described how chromospheric material is ejected into the corona, but what does the jet look like in synthetic chromospheric observations? In this subsection we show how the jet that resembles a type II spicule appears in synthetic chromospheric diagnostics. Let us begin with discussing the on-disk view and then proceed to the off-limb view.

\subsubsection{On-disk Appearance}

In observations on the disk, RBEs appear as a clearly separate component of absorption in the blue wing of the Ca II $8542 \AA$ 

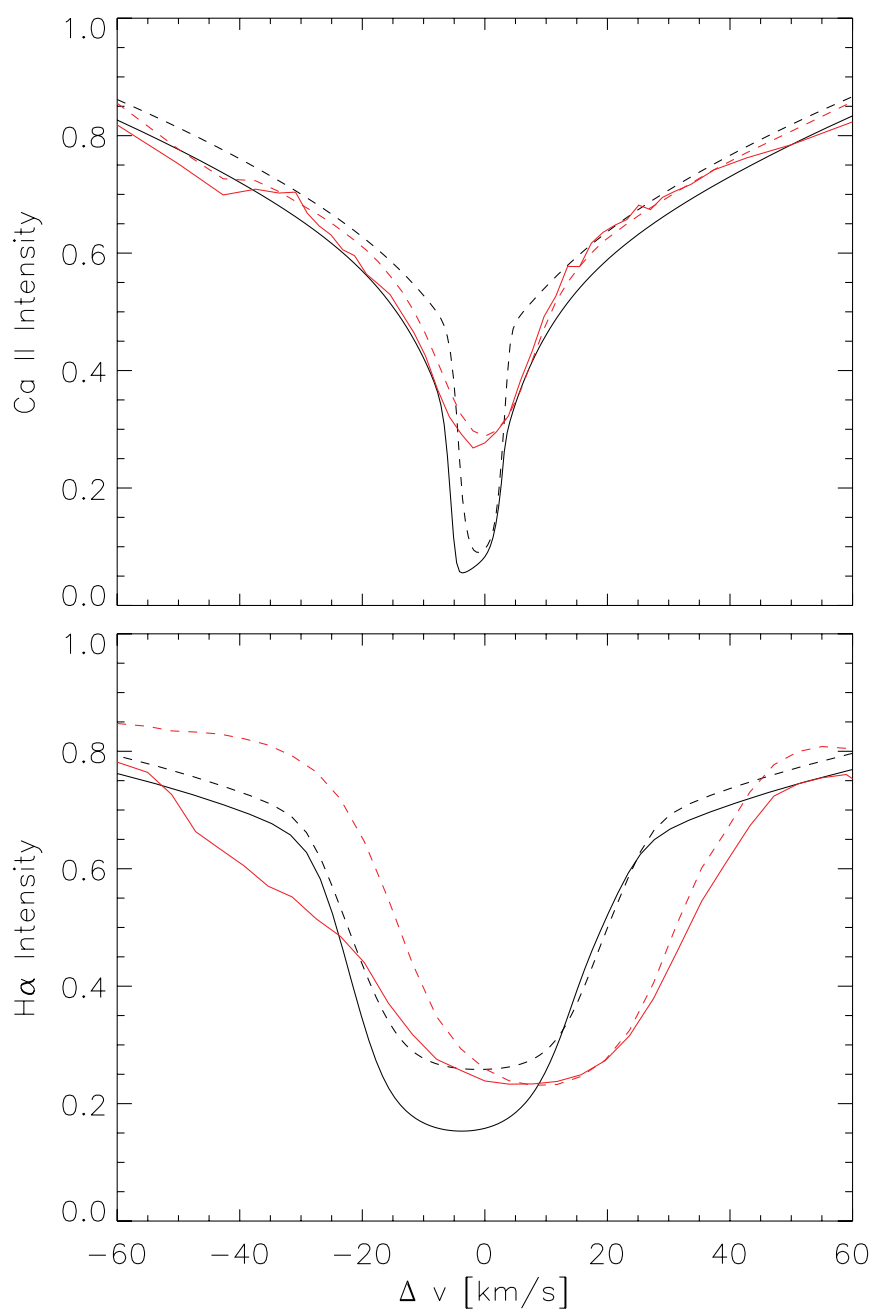

Figure 2. Comparison of observed on-disk RBEs (red curves) and the synthetic line profiles of the synthetic spicule (black curves, at simulation time $t=1590 \mathrm{~s}$, at the location of the red cross in Figure 3). The top panel shows the Ca II $8542 \AA$ line, and the bottom panel shows the $\mathrm{H} \alpha$ line. The solid curves are the line profile in the observed RBE and the synthetic spicule, the dashed curves are the average profile of a region surrounding the RBE and synthetic spicule. The intensities are normalized on the maximum intensity of the mean profile.

(A color version of this figure is available in the online journal.)

and $\mathrm{H} \alpha$ profiles (for example, see Figure 1 of Rouppe van der Voort et al. 2009). The solid red curves in Figure 2 show typical examples of RBE profiles, with a clearly lower-thanaverage intensity in the blue wing, with an unaffected red wing. In contrast, the synthetic profiles of the spicule do not show such blue-wing asymmetry. Instead both the Ca II $8542 \AA$ and $\mathrm{H} \alpha$ profiles show an asymmetric line core, whose minimum intensities are blueshifted $\sim 4$ and $\sim 5 \mathrm{~km} \mathrm{~s}^{-1}$ respectively from the rest wavelength of the line. The shift is much smaller than the upflow velocities in the simulated spicule. Figure 2 is chosen at $t=1590 \mathrm{~s}$, i.e., when the spicule reaches the longest insertion into the corona.

Figure 3 shows why the synthetic profiles do not show a blue-wing absorption coefficient. The top row shows line-center images; the location of the spicule does not stand out (this is also the case in observations). The middle row shows blue-wing images. Here the spicule location shows up as small patches of below-average intensity, but it does not stand out from the other dark structures in the image. The bottom row finally shows why the spicule does not leave an imprint in the profiles and images.
The spicule is visible as the thin cold jet protruding into the hotter coronal material. However, the overplotted optical depth curves show that the spicule has a vertical optical thickness smaller than $10^{-3}$, too small to leave an imprint on the emergent profiles: the optical thickness at $z=3 \mathrm{Mm}$ is actually only $7 \times 10^{-7}$. This lack of opacity can be caused by too low mass density and/or too high temperature that lead to ionization to Ca III and therefore too little opacity. We tested the effect of the temperature by assuming all calcium in the atmosphere is in the form of Ca II and computing the upper bound of the optical thickness of the spicule given the mass density. The spicule then gets an optical thickness at $z=3 \mathrm{Mm}$ of 0.02 , which is also too small to leave an imprint on the emergent profile. Having a lower temperature spicule (or having a delayed ionization of Ca II through non-equilibrium ionization) would thus not be enough to get an observable signal; the mass density is also too low by at least a factor of five. This is consistent with the low electron density in the simulated spicule $\left(\sim 2 \times 10^{9} \mathrm{~cm}^{-3}\right.$ above $z=3 \mathrm{Mm}$ ) compared with electron densities derived from spicule observations $\left(5 \times 10^{10}-2 \times 10^{11} \mathrm{~cm}^{-3}\right.$; Beckers 1968, 1972).

The lack of density may be caused by the fact that the spicule does not eject enough chromospheric material. It may also be that the spicule expands too fast in the corona due to the magnetic field expansion. The simulations have a rather simplified magnetic field configuration (Figure 1) compared with those on the Sun, e.g., the simulated emerging flux tube is a longitudinal flux tube along the $x$-axis. In contrast, in the Sun the small-scale emergence occurs frequently, and the orientation of these events is random Martínez-González et al. (2007). The observed field strengths are also different from those in our simulation. In addition, the modeled ambient magnetic field does not show the same salt and pepper distribution as in the solar photosphere. The simulated magnetic field distribution is most likely also not reproducing the interaction between network and internetwork and the various scales observed in the Sun. Note that the magnetic complexity seems to play a role in eruptions (see, e.g., Georgoulis et al. 2012) and it might be expected that a more complicated modeled field could lead to more violent chromospheric and coronal dynamics. This may explain why these jets are rare in the simulations, i.e., because we lack magnetic field complexity and the interaction between various magnetic features.

The cores of the synthetic profiles are narrower than those of the observed profiles (Figure 2). This discrepancy between synthetic and observed profiles exists not only in the spicule but in all current simulations. Recent analyses suggest that this is related to the relatively low spatial resolution of the simulations.

In contrast to the observations, where the spicules are usually not aligned with the LOS, the synthetic spicule is aligned with the vertical axis. First results done by Rouppe van der Voort et al. (2009) revealed that most of the observed RBEs show an acceleration along them. Most recent and more detailed studies showed that observed RBEs can show a Doppler shift increase or decrease, or a variable distribution with height (Sekse et al. 2012). In our simulated spicule, we investigate how the spicule aligned flows depend on height by calculating the velocity along an LOS that is $30 \mathrm{deg}$ off the vertical axis. Figure 4 shows this projected velocity at $t=1590 \mathrm{~s}$ for positions where the spicule is located and the temperature is lower than $4 \times 10^{4} \mathrm{~K}$. The projected velocity increases along the spicule, basically because the plasma is accelerated along the whole spicule and not at a single location. This acceleration is due to the fact that the compression 

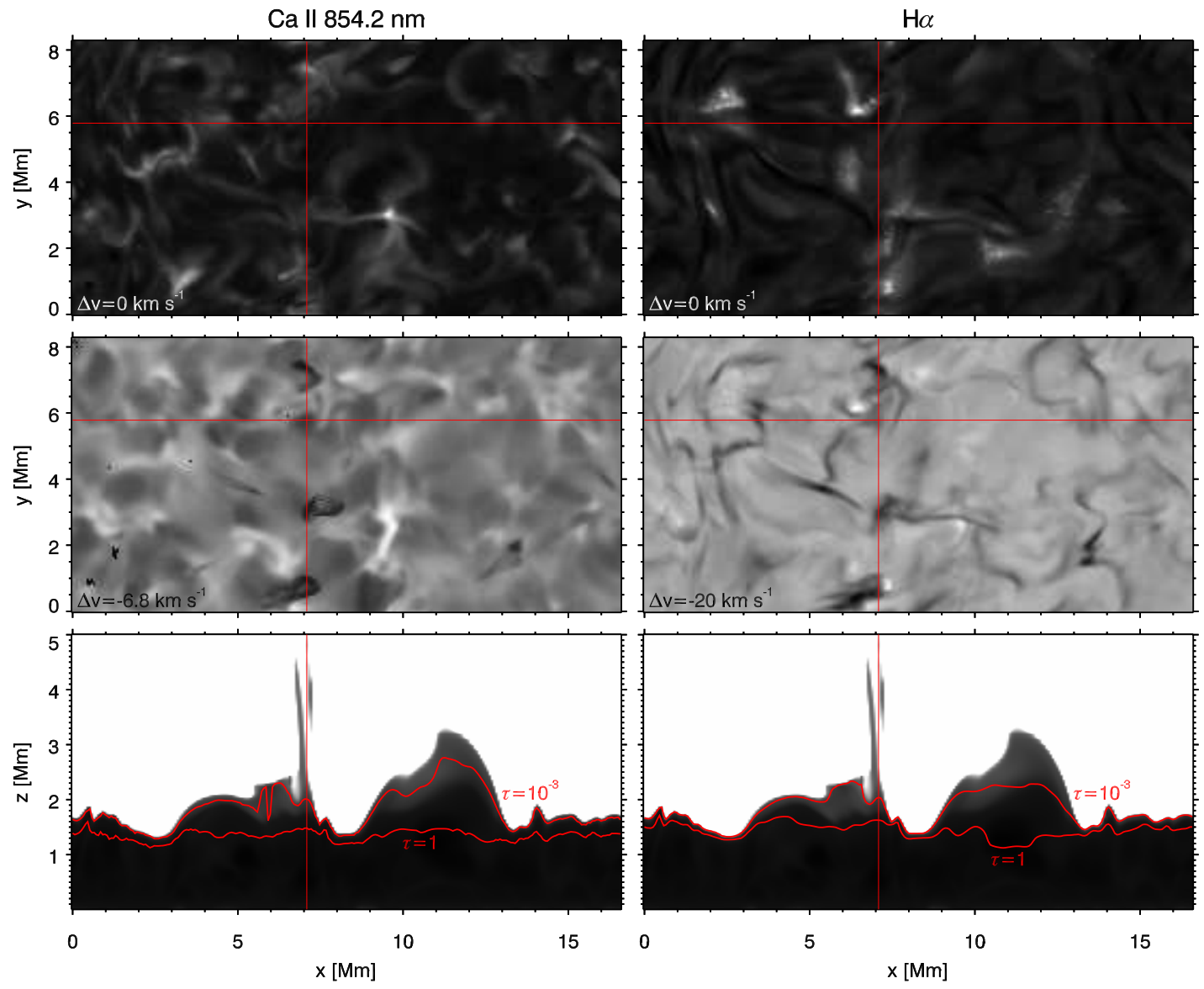

Figure 3. Images of the on-disk Ca II $8542 \AA$ (left-hand panels) and $\mathrm{H} \alpha$ intensity (right-hand panels) at line-center (top row) and in the blue wing (middle row). The Doppler shifts are indicated in the lower left corner of each panel. The red crosshairs indicate the location of the spicule and the line profile shown in Figure 2 . The bottom row shows an $x z$-slice of the temperature in the simulation (clipped at $20 \mathrm{kK}$ ) along the horizontal red line in the upper panels. The height of $\tau=10^{-3}$ and $\tau=1$ are shown as red curves. The vertical red line again indicates the location of the spicule.

(A color version of this figure is available in the online journal.)

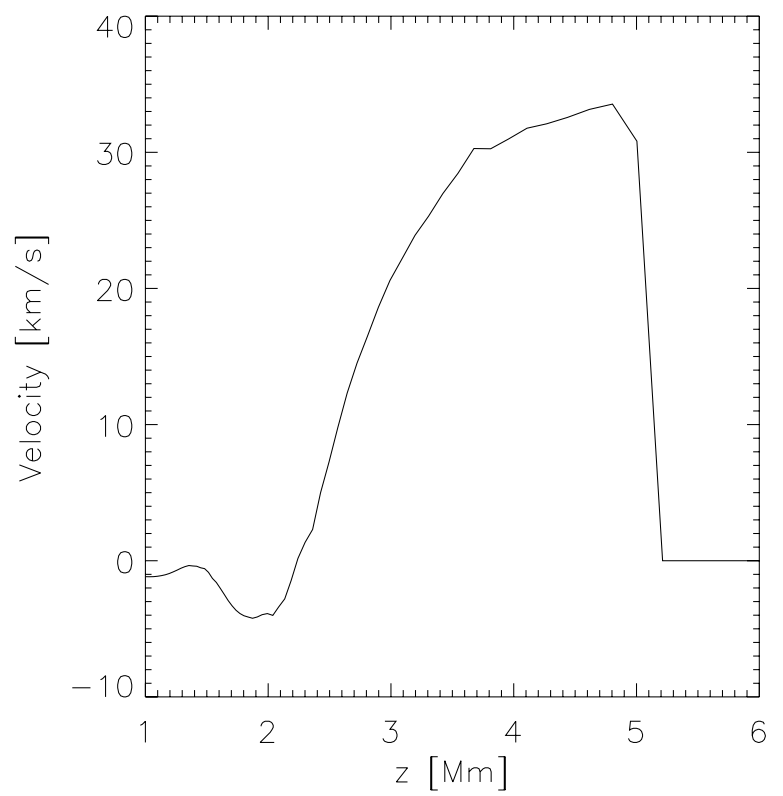

Figure 4. Increase of projected velocity along the simulated spicule. The horizontal mean of the velocity projected at an angle of $30 \mathrm{deg}$ with respect to the vertical axis is shown as a function of height at $t=1590 \mathrm{~s}$ where the spicule is located and the temperature is lower than $4 \times 10^{4} \mathrm{~K}$. This increase is similar to observations that show an increase of the Doppler shift with distance along the RBEs. acts along a large stretch along the spicule (Martínez-Sykora et al. 2011). This may explain the observed increase of Doppler shift with height along RBEs (Sekse et al. 2012).

\subsubsection{Off-limb Appearance}

Figure 5 shows images of the simulated off-limb intensity at the center of $\mathrm{H} \alpha$ (left) and Ca II $\mathrm{H} 3968 \AA$ (middle) as well as a $z t$-slice of $\mathrm{Ca}$ II $\mathrm{H}$ (right). The intensity was computed by solving the transfer equation along the $y$-axis for each $x z$ point in the simulation box. The effect of the curvature of the Sun was not taken into account. The spicule reaches heights up to $4.9 \mathrm{Mm}$ for $\mathrm{Ca}$ II $\mathrm{H}$ and $5.5 \mathrm{Mm}$ for $\mathrm{H} \alpha$ above the photosphere; i.e., this is a small type II spicule but still within their observed height range (Pereira et al. 2012). Note that in the spicule the synthetic $\mathrm{H} \alpha$ emission extends to a larger heights $(\sim 0.6 \mathrm{Mm})$ than the $\mathrm{Ca}$ II $\mathrm{H}$ emission. Even though this is at the limb and for a different $\mathrm{Ca}$ II line, it seems compatible with recent observations where the location of Ca II $8542 \AA$ RBEs are found closer to the footpoints than $\mathrm{H} \alpha$ RBEs (Rouppe van der Voort et al. 2009; Sekse et al. 2012). The synthetic spicule also shows substructure suggestive of multiple threads, consistent with some observations of offlimb spicules with Hinode (Suematsu et al. 2008). This structure is caused by the density and temperature variation within the spicule. This variation is caused by two factors: (1) the energy release is not uniformly distributed across and along the spicule; and (2) the ejected plasma does not uniformly expand into the 


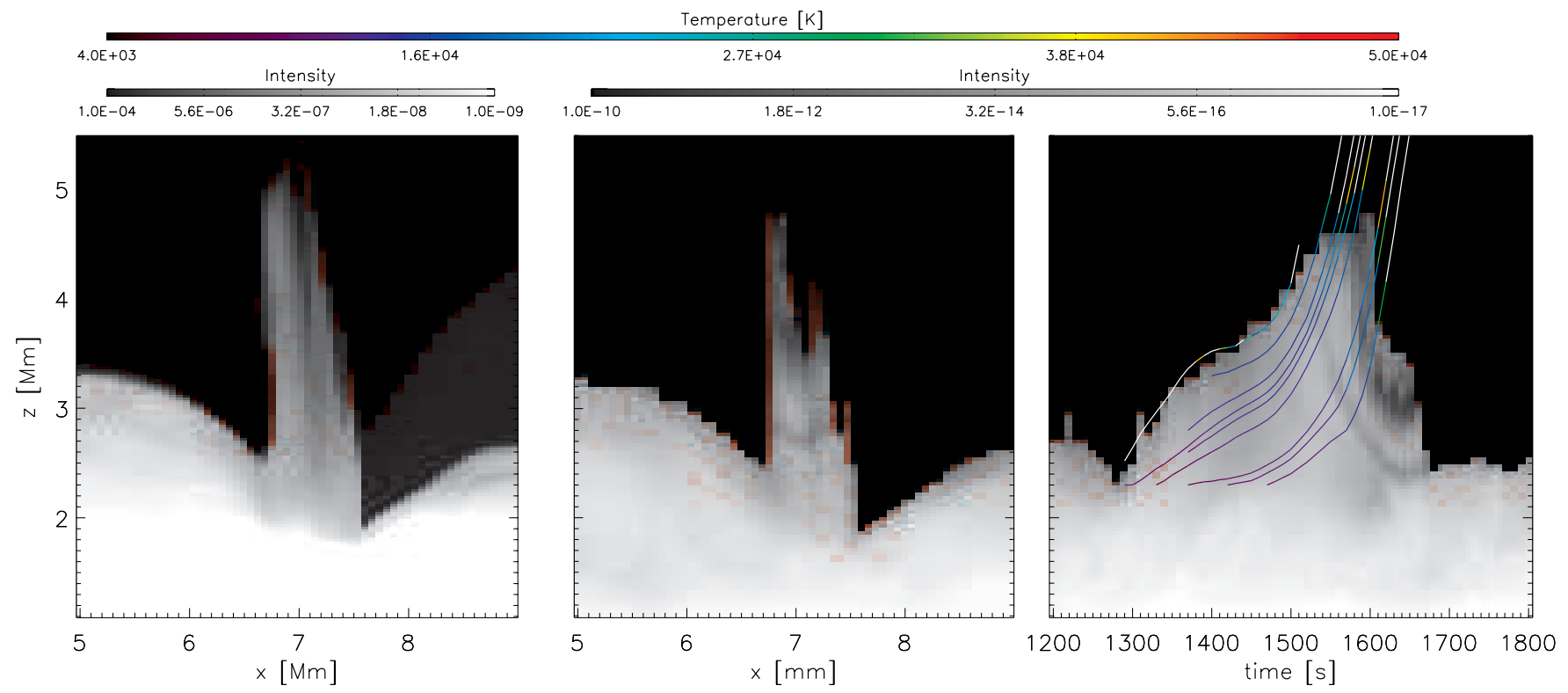

Figure 5. Appearance of the synthetic spicule in chromospheric lines at the limb. $\mathrm{H} \alpha$ and Ca II H $3968 \AA$ Antensity from side-view (left and middle panels, respectively) at $t=1590 \mathrm{~s}$ and Ca II $\mathrm{H}$ intensity and as a function of time and height at $x=[6.7] \pm 0.2 \mathrm{Mm}$ (right panel). The intensity is shown on a logarithmic scale. The right panel also displays a number of test particle trajectories to indicate the matter flow. The trajectories are color coded with the gas temperature.

(A color version of this figure is available in the online journal.)

corona because the magnetic field lines do not uniformly expand with height.

One of the major discrepancies between the off-limb observations and synthetic observables is the difference in scale height intensity for the line core emission of $\mathrm{Ca}$ II $\mathrm{H}$ and $\mathrm{H} \alpha$. At $2.2 \mathrm{Mm}$ above the limb of the quiet Sun, Pereira et al. (2012) find a scale height of 2-3 Mm in the $\mathrm{Ca}$ II $\mathrm{H}$ line. In contrast, in the synthetic observables in the vicinity and inside of our spicule event, we find intensity scale heights of only $0.25 \mathrm{Mm}$ for the Ca II $\mathrm{H}$ line and $0.6 \mathrm{Mm}$ for $\mathrm{H} \alpha$. Farther from the spicule, in regions where the atmosphere is not affected by flux emergence and the spicule event, the intensity scale heights are even smaller; $0.15 \mathrm{Mm}$ and $0.25 \mathrm{Mm}$ for $\mathrm{Ca}$ II $\mathrm{H}$ and $\mathrm{H} \alpha$, respectively (the presence of flux emergence increases the intensity scale height as suggested by Martínez-Sykora et al. 2009).

As a result, the optical thickness at line center of the $\mathrm{H} \alpha$ spicule at $z=4 \mathrm{Mm}$ is $3 \times 10^{-5}$, i.e., this signal may be too faint to detect in real observations. We speculate that there are several reasons for the difference in intensity scale height.

1. As a result of the small size of the numerical domain, the simulation lacks the LOS superposition of background intensity at spicular heights.

2. The simulation likely has a spatial resolution that is too low.

3. As mentioned in Section 4.1.1, the spicule does not eject enough chromospheric material and/or expands too quickly into the corona.

4. As alluded, the simulation does not mimic emergence of small-scale flux on the Sun, i.e., the salt and pepper distribution and the small-scale flux emergence distribution in the simulation do not reproduce the observations well.

5. Finally, current calculations do not take into account timedependent ionization. The latter effect can in principle lead to larger formation heights for both $\mathrm{Ca}$ II $\mathrm{H}$ and $\mathrm{H} \alpha$ (Wedemeyer-Böhm \& Carlsson 2011; Leenaarts et al. 2007).

At the limb the spicule shown in Ca II H (right panel of Figure 5) rises $1 \mathrm{Mm}$ in 100 s, i.e., the apparent upward speed is around $10 \mathrm{~km} \mathrm{~s}^{-1}$. This speed is significantly smaller than the observed apparent velocities in Ca II $\mathrm{H}$ filtergrams at the limb (Pereira et al. 2012). In the early state of the spicule, the leftmost streamline indicates that spicular material at the top of the spicule rises with the same speed as the apparent upward motion. Spicular material that is accelerated upward at later times reaches larger velocities, up to $40 \mathrm{~km} \mathrm{~s}^{-1}$. The apparent upward motion of the off-limb emission is stopped as the gas in the spicule is heated to temperatures above $20 \mathrm{kK}$ and all calcium is ionized to Ca III. Therefore, the apparent acceleration in the second half of its evolution is limited by the ionization front. This process is similar to what Heristchi \& Mouradian (1992) suggested. The spicule then fades rapidly from view in Ca II $\mathrm{H}$ intensity. The spicule does not vanish because the plasma descends but because calcium is ionized away.

\subsubsection{Heating of the Spicule Out of the Chromospheric Line Passbands}

Figure 6 shows the various heating and cooling sources per particle in the spicule. The dominant heating mechanism is the Joule heating. However, the complexity of the spicule shows that other sources also can play a role in different parts of the spicule. For example, thermal conduction seems to be rather important in regions where the temperature of the plasma reaches $\sim 10^{5} \mathrm{~K}$. These regions are located at the edge of the chromospheric material; the upward flowing plasma that stays cool the longest and that penetrates into the corona is finally heated by conduction (this parcel of plasma can be appreciated in the running time difference of He II $304 \AA$ shown in Figure 8 around $z \sim 6 \mathrm{Mm}$ and $t=1650 \mathrm{~s}$ ).

Inside the chromospheric material of the spicule, the radiative cooling (panel (E) in Figure 6) plays an important role counteracting the viscous and Joule heating (panels (A) and $(\mathrm{F})$ ). The radiative cooling is greater in the earlier states of the spicule. When the spicule expands into the corona, the density drops, and the Joule heating also becomes more important: the chromospheric material heats up with time and radiative losses 


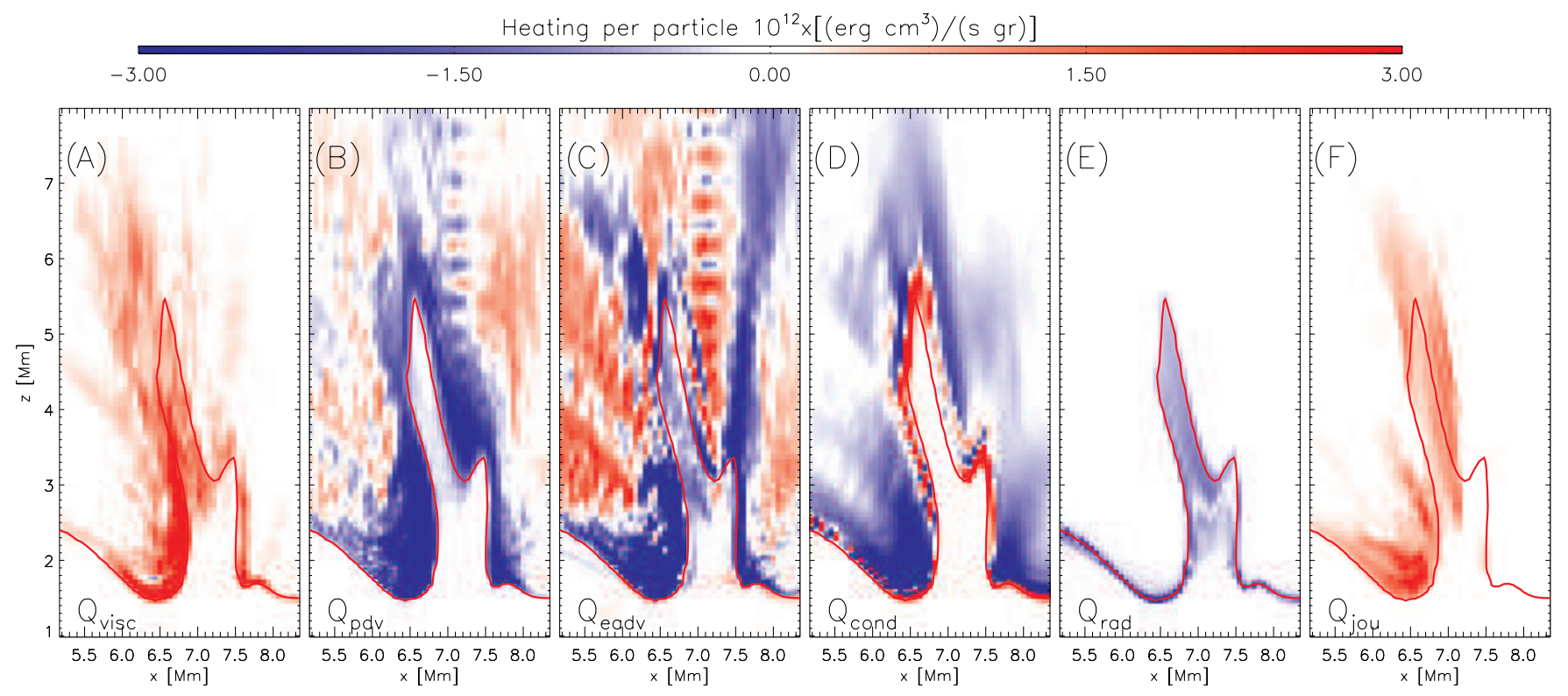

Figure 6. Various heating and cooling sources are shown in the top row, i.e., viscous heating (panel (A)), compression work $(P \nabla u)($ panel $(B))$, advection (panel (C)), conduction (panel (D)), the radiative term (panel (E)), and the joule heating (panel (F)). The vertical cut is at $y=6.175 \mathrm{Mm}$, at $t=1590 \mathrm{~s}$. The temperature at $2 \times 10^{5} \mathrm{~K}$ is overlaid on the plots with red contours.

(A color version of this figure is available in the online journal.)

decrease. The largest contributions to the radiative cooling come from hydrogen and calcium ions.

Finally, one of the hot footpoints is heated mainly due to Joule heating (Figure 6). Afterward, conduction (panel (D)) and advection (panel (C)) spread this heat along field lines. As can be appreciated in the figure, the regions where the different heating sources are concentrated are not easily linked, and the 3D structures are rather complex. We would like to remark that, in contrast, type I spicules do not have strong sources of Joule heating, and the plasma suffers an expansion followed by a contraction (Hansteen et al. 2006; MartínezSykora et al. 2009). Therefore, this type of spicules shows the well-known parabolic profile. However, the type II spicule candidate shows a complex contribution of various heating and cooling terms, emphasizing the role of Joule heating. In addition, the chromospheric plasma does not experience an expansion followed by a compression, but it is ejected into the corona and heated.

We are neglecting physical processes in the chromosphere that may impact the evolution of the spicule:

1. The ion-neutral effects such as a proper treatment of Generalized Ohm's law play an important role that may change the way the spicule is heated (see a discussion of the importance of these effects in Martínez-Sykora et al. 2012).

2. Joule heating in the model is dependent on numerical dissipation in order to keep structures resolvable on the numerical grid, therefore they will occur on much larger scales than those found on the Sun. This may have important implications for the details of type II spicule heating.

3. Ionization of hydrogen and helium is treated with the simplifying assumption of LTE. Proper inclusion of nonequilibrium ionization (Leenaarts et al. 2007, 2011) will lead to a different response of the thermodynamic state of the atmosphere to sudden heating.

\subsection{Emission in EUV, from Transition Region to Coronal Lines: Impact into the Corona}

We study the impact of the simulated type II spicule on the corona using synthetic EUV intensities and spectra under the optically thin approximation and ionization equilibrium conditions as described in Section 2.2.

\subsubsection{Intensity: Heating and Cooling Information}

During the evolution of the spicule, it shows considerable emission in transition region and coronal EUV lines in several different ions. Figure 7 shows the intensity from the top and limb view coming from the ions He II $304 \AA$, Fe IX $171 \AA$, and Fe XIV $211 \AA$ A using full numerical spatial resolution (top) and convolved to a Gaussian with the same width as the SDO/AIA point-spread function and rebinned to its spatial resolution (bottom panels). These lines constitute the strongest contributions to the $304 \AA, 171 \AA$, and $211 \AA S D O /$ AIA channels, respectively (the same channels were analyzed by De Pontieu et al. 2011, to show the emission of the type II spicules in the corona). We consider only the dominant ions to isolate the information of the specific SDO/AIA channels from the emission caused by other "non-dominant" lines (Martínez-Sykora et al. 2011b). It is important to mention that He II $304 \AA$ Alne formation is poorly understood, so it is not clear that the coronal approximation approach is valid (Feldman et al. 2010). Nevertheless, we calculated the line assuming coronal approximation and that the line is optically thin.

It is interesting that the locations of the various emissions are not co-spatial due to the complex thermal process occurring inside and surroundings the spicule. For instance, the He II $304 \mathrm{~A}$ emission at the limb clearly shows the structure of the spicule, i.e., the shape of the ejected chromospheric material, but most of the emission coming from Fe IX $171 \AA$ is concentrated at the footpoint of the spicule, and for Fe XIV $211 \AA$ it is also along field lines that connect to the footpoints of the spicule where most of the heating source is located. 

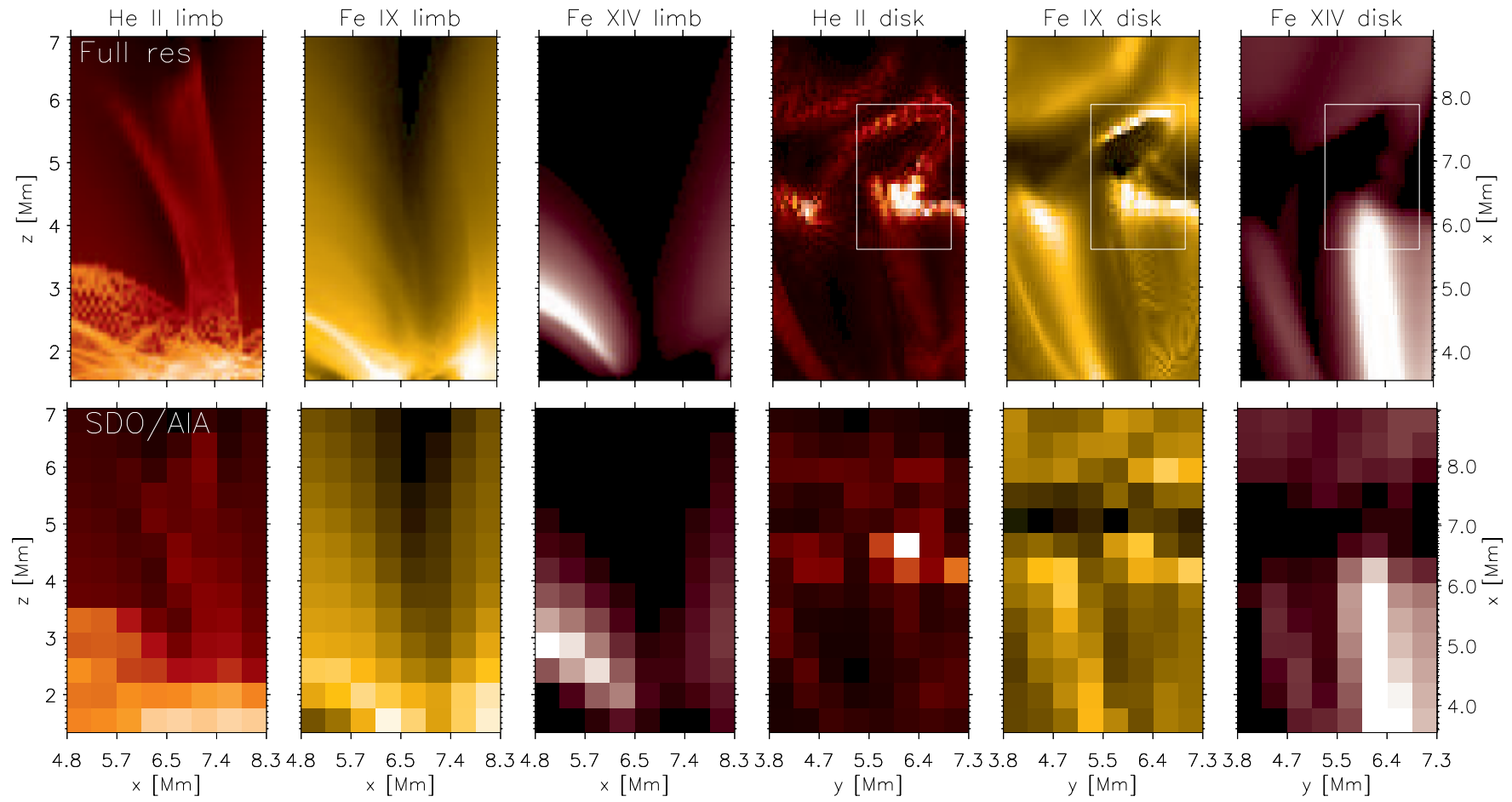

$\times[\mathrm{Mm}]$

$\times[\mathrm{Mm}]$

$\times[\mathrm{Mm}]$

$4.7 \quad 5.5 \quad 6.4$
$y[\mathrm{Mm}]$

$\begin{array}{ll} & 5.5 \\ y & {[\mathrm{Mm}]}\end{array}$

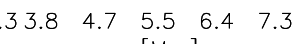

Figure 7. Synthetic intensity maps at time $t=1630$ s for He II $304 \AA$ (first and fourth columns), Fe IX $171 \AA$ (second and fifth columns), and Fe XIV $211 \AA$ (third and sixth columns) for a limb view (first three columns) and top view (last three columns). The top row is at the spatial resolution of the simulation and the bottom row is taking into account the SDO/AIA spatial resolution.

(A color version of this figure is available in the online journal.)

In the disk view, the emission coming from He II $304 \AA$ is not concentrated where the spicule is, but at the side boundaries of the ejected chromospheric material, rather similar to Fe IX $171 \AA$ A. This is basically because at the footpoints of the spicule the density is higher than at the top of the spicule, and both the He II $304 \AA$ and Fe Ix $171 \AA$ contribution functions peak near the transition region, i.e., surrounding the spicule. The spicule is heated but the plasma inside the spicule is never "filled in" with the emission of these lines because the spicule is not heated uniformly. In contrast, the emission coming from Fe XIV $211 \AA$ is located along the field lines that connect to the footpoint of the spicule. As a result, it is a significant challenge to link the emission coming from the different ions in space and time. Note that some of the detailed spatial structuring of the spicules observed in the different lines vanishes when taking into account $S D O /$ AIA spatial resolution.

Disentangling emission from various ions in the spicule is difficult because of the complexity of the simulated jet. An additional complication, arises from the fact that the various SDO/AIA channels have contributions from other, typically non-dominant lines (Martínez-Sykora et al. 2011b, studied these contributions in 3D MHD simulations). We find that the nondominant lines do not play a significant role in the emission of the simulated spicule. We note that the most significant nondominant lines in the 171, 193, and $211 \AA$ SDO/AIA channels are emitted by ions formed at very similar low transition region temperatures (e.g., O v/vI). This means that significant emission from such ions in spicules would occur at the same location and time for the various SDO/AIA channels. We can exploit this to distinguish between emission coming from the dominant ion and the non-dominant ions. As we can see in Section 4.3 our model agrees well with the observed offset space and time in the SDO/AIA channels (De Pontieu et al. 2011). This supports the hypothesis that the spicule emission in the various $S D O /$ AIA channels is not a low temperature contamination as suggested by Madjarska et al. (2011).

Figure 8 shows the intensity from the top-view (left two columns) and a limb view (right two columns) as a function of time and length along the spicule coming from He II $304 \AA$ (top row), Fe IX $171 \AA$ (second row), Fe XII $193 \AA$ (third row), and Fe XIV $211 \AA$ (last row). The running differences are shown in the second and last columns. These lines correspond to the strongest contribution of the 304, 171, 193, and $211 \AA$ SDO/AIA channels, respectively. The images on the disk (two left columns) show that all channels show strong emission for a relatively long time period. In fact, the emission increases in time over more than 5 minutes. This increase starts earlier in cooler lines such as He II $304 \AA$ and Fe IX $171 \AA$. In addition, a weak brightening on the disk for the various filters is formed in the spicule when it is ejected $(t \sim 1550 \mathrm{~s})$, but it is very faint and it can be appreciated only in the running difference. Additionally, the increase is fainter for hotter lines (compare with observations, Section 4.3). It is also interesting that on the disk He II $304 \AA$ is concentrated in a small region, but for the coronal lines the emission is spread out over more than $2 \mathrm{Mm}$. Note that the various plots in Figure 8 do not follow a loop but are straight lines. In fact for Fe IX $171 \AA$, Fe XII $193 \AA$, and Fe XIV $211 \AA$ the source of this strong emission comes from the footpoint of the spicule, which is heated by Joule heating (see at $x=6.2$ and $z=2.2 \mathrm{Mm}$ in Figure 6) and expanded along the field lines by conduction.

At the limb, a weak signal can be seen also in Fe XII $193 \AA$ and Fe XIV $211 \AA$ after the spicule is ejected. The type II spicule candidate starts to appear at the side view around $t=1400 \mathrm{~s}$. The coronal lines seems to agree with the observations of De Pontieu et al. (2011). The emission moves into the corona 

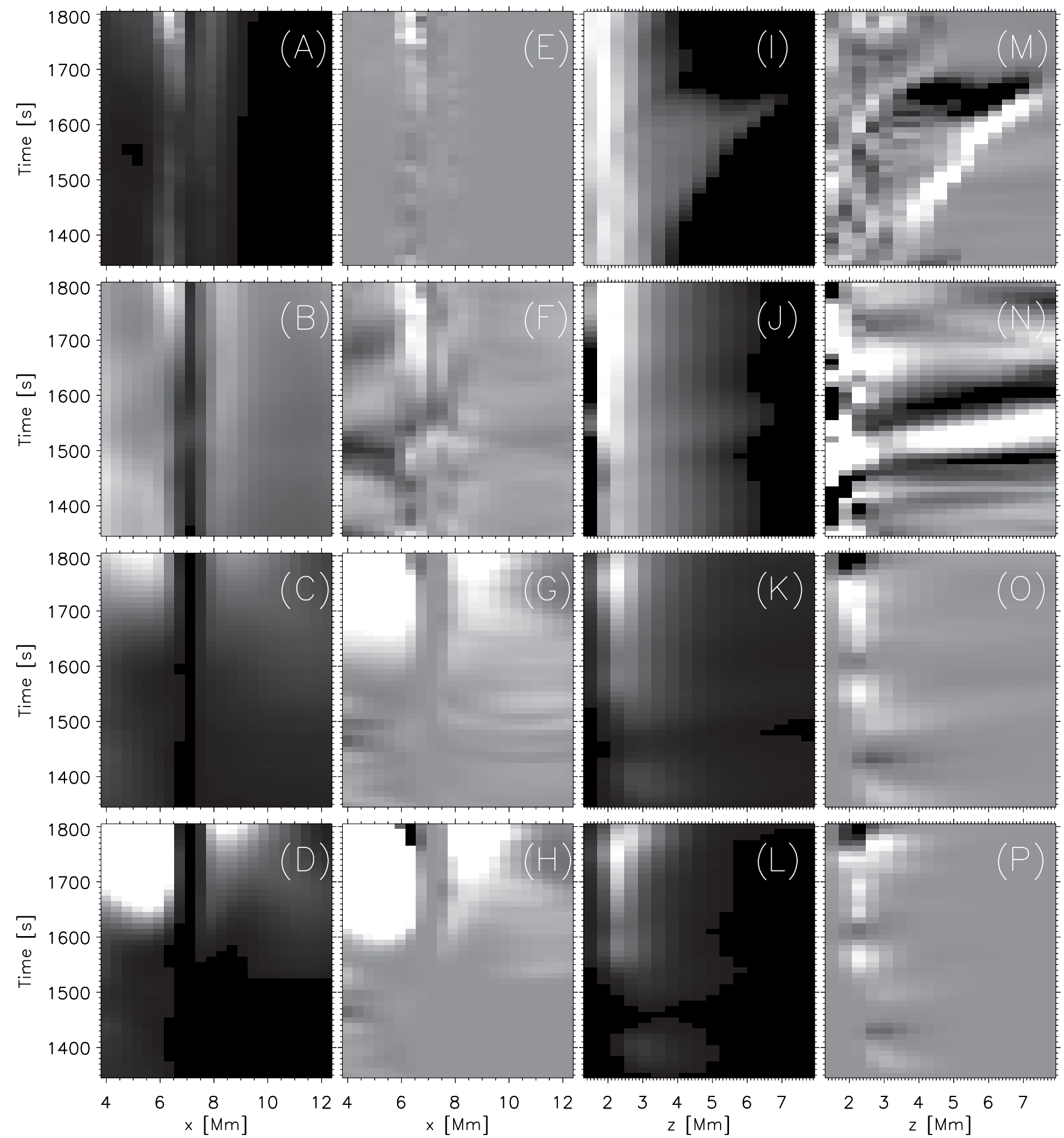

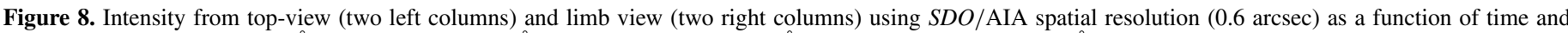

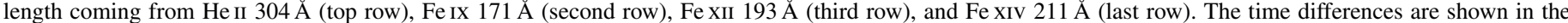

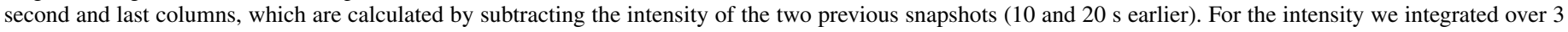
pixels in the $y$-direction for the on-disk observations and in the $x$-direction for the limb observations.

as a propagating coronal disturbance at $\sim 100 \mathrm{~km} \mathrm{~s}^{-1}$. Note that the plasma does not travel at that speed, i.e., the apparent propagation of the emission is a combination of mass flow, waves, and thermal conduction front along the field lines. The plasma flow is sonic up to temperatures $8 \times 10^{5} \mathrm{~K}$ and subsonic at higher temperatures (Martínez-Sykora et al. 2011). The strongest signal is from Fe IX $171 \AA$, but is also noticeable in Fe XII $193 \AA$ and Fe XIV $211 \AA$. Nevertheless, the strongest contribution of these two lines comes roughly one hundred seconds later. In a similar fashion as seen on the disk, for Fe IX $171 \AA$, Fe XII $193 \AA$, and Fe XIV $211 \AA$ the source of this strong emission comes from the footpoint of the spicule, which is heated by Joule heating and propagated along the field lines by conduction into the upper layers in the corona. In contrast, the formation temperature of He II $304 \AA$ is too low for the conduction. However, thermal conduction plays a role in the 


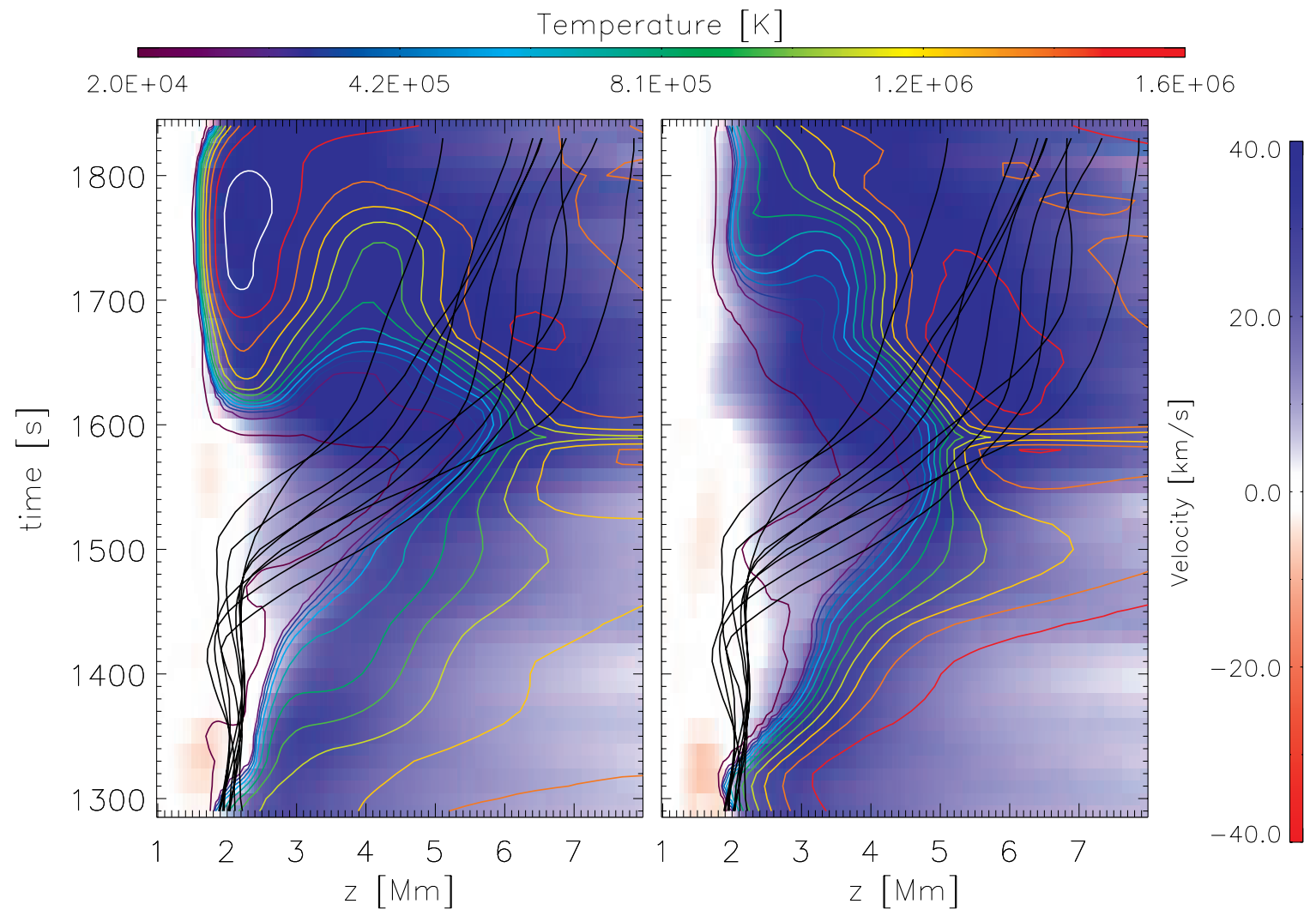

Figure 9. Vertical velocity (red-white-blue background color scheme) and temperature (color contours) are shown along a vertical axis centered in $y=6 \mathrm{Mm}$ and $x=7.02 \mathrm{Mm}$ (left panel) and $x=6.83 \mathrm{Mm}$ (right panel). The projection of particle trajectories are shown with black lines.

(A color version of this figure is available in the online journal.)

fading in the He II $304 \AA$ A emission around $t=1650 \mathrm{~s}$ : as the last remaining chromospheric parcel penetrates into the corona, the plasma around $10^{5} \mathrm{~K}$ is heated beyond the He II $304 \AA$ formation temperature (see panel (M) around $z \sim 6 \mathrm{Mm}$ and $t=1650 \mathrm{~s}$ in Figure 8). We note the lifetime of the profiles in He II $304 \AA$ is of the same order as in the observations (a few hundred seconds). Compare these results with the observations described in Section 4.3.

It is important to mention that the length, "propagation" and duration of the emission of Fe IX $171 \AA$, Fe XII $193 \AA$, and Fe XIV $211 \AA$ are strongly dependent on the magnetic field configuration. In fact, our domain is small and most of the magnetic field lines are confined within the small domain with only a few open field lines that cross the top boundary. Plasma on the latter loses heat because the upper boundary is open. As a result of this, the open field lines do not maintain the high temperatures because the energy is transported through the top of the domain due to thermal conduction. In contrast, the closed field lines produce small hot loops that are heated by the Joule heating coming from the footpoint of the spicule. This magnetic field configuration differs from that usually found on the Sun (Section 4.3). The field lines in the Sun are probably more complex and, e.g., in plage regions the magnetic field lines connect over very large distances, so that heat can propagate farther and be distributed to greater distances. In a similar manner, the magnetic field configuration of our simulation is also completely different from the magnetic field in coronal holes. The field configuration of the simulation, despite the simplicity of the magnetic field configuration and the small size, which forces it to have small loop structures in the corona, may nevertheless be "similar" to a quiet Sun.
At the limb, the emission in He II $304 \AA$ A shows a feature rising in a similar fashion as in $\mathrm{Ca}$ II $\mathrm{H}$, but it reaches greater heights (almost $2 \mathrm{Mm}$ higher; see Figure 8). However, the synthetic He II $304 \AA$ disappears from the limb view in a different fashion than $\mathrm{Ca}$ II $\mathrm{H}$. The latter disappears in a few seconds because the plasma is heated out of the passband. The synthetic He II $304 \AA$ at the limb does not vanish as fast as the synthetic Ca II $\mathrm{H}$.

The synthetic He II $304 \AA$ shows that the upper part of the spicule penetrates into the corona and at the same time fades until it disappears. While the typical observed behavior is for He II $304 \AA$ spicules to follow a parabolic profile with time (De Pontieu et al. 2011), a few examples in Figure S5 in De Pontieu et al. (2011) actually show similar behavior to our simulation: e.g., at height $5 \mathrm{Mm}$ and $t=2200 \mathrm{~s}, t=2800 \mathrm{~s}$, and $t=3800 \mathrm{~s}$. The reason for this evolution in our simulation is because the upper part of the spicule is gradually heated from above by Joule heating and conduction (see left panel of Figure 9 and right panel of Figure 5).

The observed parabolic height-time profile of He II $304 \AA$ type II spicules has typically been interpreted as a consequence of plasma moving up and down, similar to the cooler dynamic fibrils and type I spicules (Hansteen et al. 2006; De Pontieu et al. 2007a; Heggland et al. 2007). Our simulation suggests another possible scenario where a combination of mass flows, thermal evolution, and narrow imaging passbands produce apparent up and down motion. In the right panel of Figure 9 the temperature contour around the formation temperature of He II $304 \AA$ (violet contours) appears to follow some sort of parabolic profile. When the spicule reaches its greatest height, the spicule starts to fade, which then causes the parabolic shape for transition region temperatures seen in space-time plots of intensity similar to the 

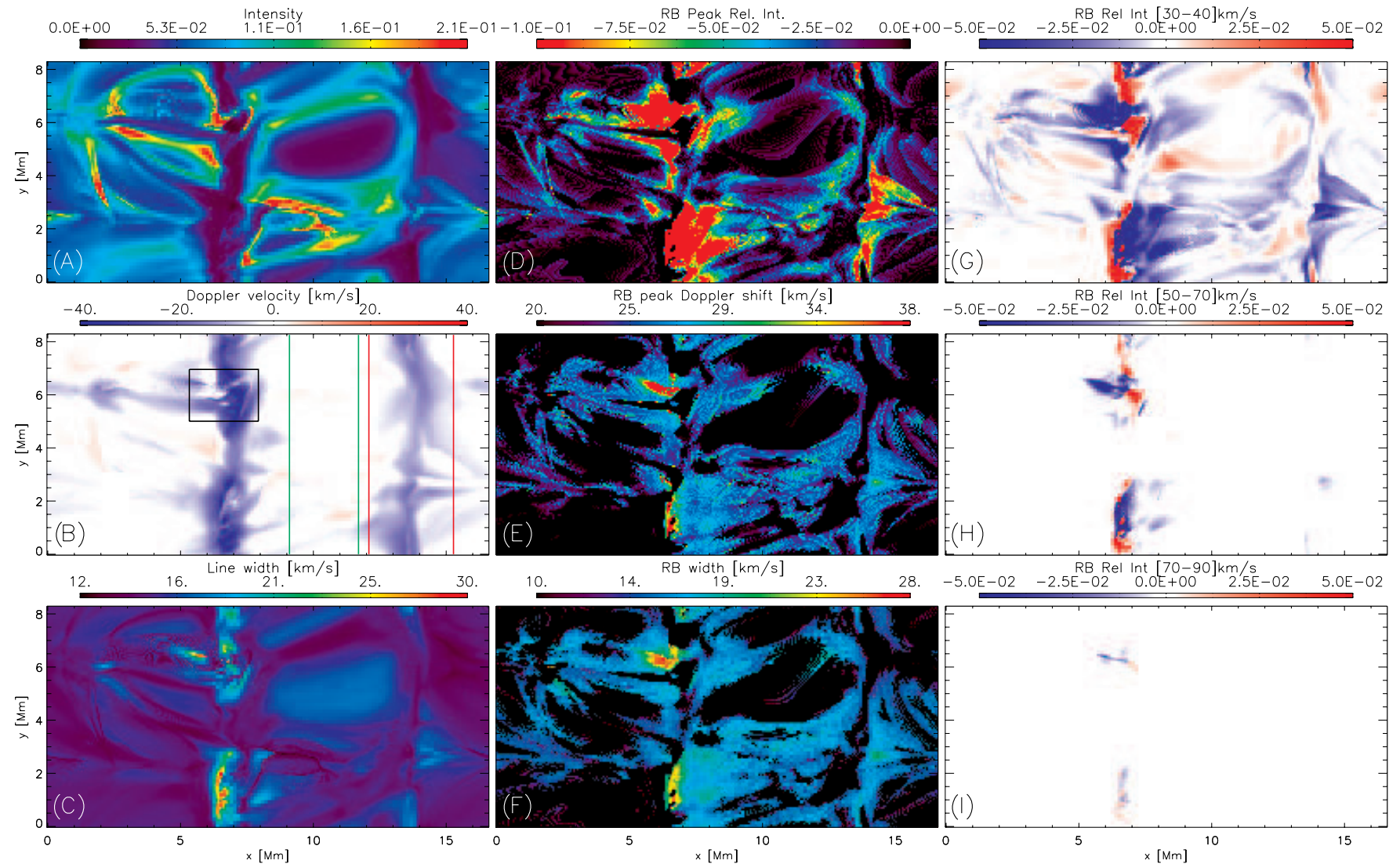

Figure 10. Spatial distributions of the parameters derived from the single-Gaussian fit and RB asymmetry analyses for Si VII $275 \AA$ at $t=1610 \mathrm{~s}$. The figure shows maps of the total intensity (panel A), Doppler velocity (panel B), line width (panel C), peak RB asymmetry (panel D), Doppler shifts of the RB peak asymmetry (panel E), width of the RB asymmetry (panel F), and RB asymmetry between [30, 50], [50, 70], and [70, 90] km s$~^{-1}$ (panels G-I). The spicule is located inside the black box in panel B, the area between the red lines delimits the footpoint of the transition region without any spicule and the area between the green lines delimits a loop region where the magnetic field is rather horizontal.

rapid fading of $\mathrm{H} \alpha$ or $\mathrm{Ca}$ II $\mathrm{H}$ described above. The heating for transition region temperatures is concentrated above the spicule and is not as rapid as in the location of the spicule shown in the left panel in Figure 9. The left and right panels are for two different locations in the spicule.

For the other EUV lines, the emission follows the field lines forming the small loop as shown in Figure 7. The length of the synthetic spicule is a bit shorter compared with the real observations (De Pontieu et al. 2011), probably because of the following.

1. The candidate presented here is not violent enough.

2. He II $304 \AA$ ionization may be time-dependent.

3 . Note that as a result of the small box size: the magnetic field lines are confined in the small domain, and we do not have the same background contribution as in the observations, i.e., we lack the large LOS integration at the limb that we find on the Sun.

\subsubsection{Line Width, Doppler Shifts, and Asymmetries}

4.2.2.1. On the disk. The ejected chromospheric material reaches velocities up to $40 \mathrm{~km} \mathrm{~s}^{-1}$, but at higher temperatures the plasma reaches larger and a wider range of upflow velocities (as shown in Figure 3 in Martínez-Sykora et al. 2011). A good diagnostic for analyzing these velocities is combining the information of the Doppler shift, line width, and RB asymmetry as shown in Figure 10 for the spectral line Si VII $275 \AA$. The spicule is located at $[x, y] \approx[6,6] \mathrm{Mm}$. It shows Doppler shifts up to $50 \mathrm{~km} \mathrm{~s}^{-1}$ (panel (B)). The line width is larger than in the surroundings of the spicule and the profile shows a strong blueward asymmetry. Both the broad line and blueward asymmetry are a direct consequence of the strong spatial variation of the LOS velocity of the plasma that emits in this spectral line. In particular, in the spicule the line width is approximately $32 \mathrm{~km} \mathrm{~s}^{-1}$. In contrast, the line width in the surroundings is only $18 \mathrm{~km} \mathrm{~s}^{-1}$ or less (panel (C)). The spicule shows blueward asymmetries of roughly $10 \%$ (panel (D)) of the intensity centered at $50 \mathrm{~km} \mathrm{~s}^{-1}$ (panel (E)). However, this blueward asymmetry is observed all the way up to $90 \mathrm{~km} \mathrm{~s}^{-1}$ (see panels $(\mathrm{G})-(\mathrm{I})$ ).

It is important to consider the instrumental effects on the spectral line, such as the spatial resolution and the instrumental broadening shown in Figure 11. Martínez-Sykora et al. (2011a) described in detail the impact of these effects on the RB asymmetry diagnostics. As a result of the spatial resolution, the Doppler shifts are significantly smaller $\left(\sim 25 \mathrm{~km} \mathrm{~s}^{-1}\right.$ for the spicule, panel (B)) because the profile is convolved with the pixel size. In general, the line width is larger due to the instrumental broadening (panel (C)). However, at the location of the spicule, the line width is even larger. In addition, the RB asymmetry profile is shifted to higher velocities (panel (E) and panels $(\mathrm{G})-(\mathrm{I})$ ), and becomes wider (panel (F)). As result of the spatial averaging, the profiles include contributions from cells in the domain, which leads to a wider distribution of velocity and temperature. In the spicule this leads to strong blueward asymmetries (see below).

In a similar manner as for Si vII $275 \AA$, transition region lines (see Figure 12 for Nv $1238 \AA$ ) and coronal lines (see 

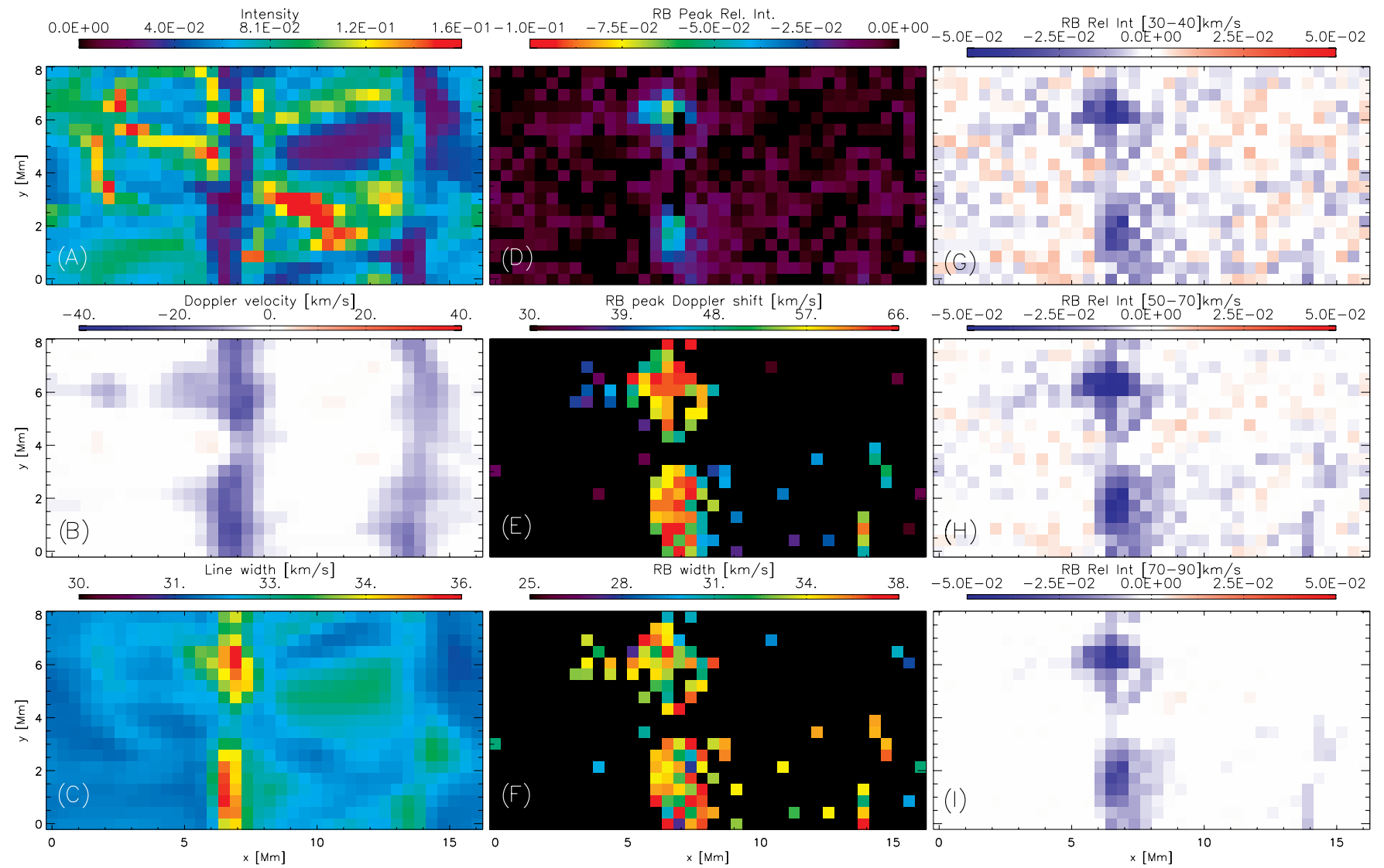

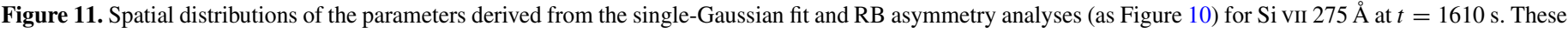
profiles are calculated taking into account the Hinode spatial resolution and instrumental broadening of spectral lines.

(A color version of this figure is available in the online journal.)

Figure 13 for Fe x $184 \AA$ A) show strong Doppler shifts, large line widths, and blueward asymmetries of the order of $5 \%$ located at $\sim 50 \mathrm{~km} \mathrm{~s}^{-1}$ at the spicule location. In the area of the spicule, the Doppler shift and asymmetry is a bit larger in Si VII $275 \AA$ than in the other two lines. This is because of the thermodynamic properties of the spicule at that instant, which changes in time as described below. In addition, Si vII $275 \AA$ is emitted by a lithium-like ion. The atomic physics of the lithium-like ions cause emission over a larger range of temperatures than for non-lithium-like ions (see below and in Martínez-Sykora et al. 2011a).

At the footpoints of the region without type II spicules (red square in panel (B) in Figure 10), the Doppler velocity increases with the formation temperature of the various ions (red in the top panel of Figure 14). However, in the loop regions (the region delimited within the red square in panel (B) in Figure 10) the Doppler velocity does not increase with the formation temperature of the ion(s) mainly because the plasma is confined to the magnetic field and it is mostly horizontal (green in the top panel of Figure 14). In the spicule, the Doppler velocities of all spectral lines (shown black in the top panel of Figure 14) are higher than the Doppler velocities in the other selected regions. The Doppler velocity seems rather constant with formation temperature of the ions for transition region lines (C IV $195 \AA$, N v $1238 \AA$, and O VI $195 \AA$ ), but for lines with higher formation temperatures the Doppler velocity increases significantly.

Despite this significant rise of Doppler shifts with temperature, the actual vertical velocity in the spicule for the temperatures covered does not increase significantly with temperature. At coronal temperatures the spicular plasma at $t=1600$ s shows only a small increase of the actual upflow velocity (as opposed to the Doppler shift, which is a property of the emergent line profile) with temperature as shown in Figure 3 in MartínezSykora et al. (2011). The modest increase in vertical velocity with temperature is considerably lower than what is expected from 1D models (Judge et al. 2012). There are several reasons for this: our simulated spicule arises as the result of Lorentz force driven acceleration that occurs along a large height range and acts differently for various locations within the jet. This complex and dynamic 3D structure defies simplification to the seemingly intuitive 1D interpretation. In addition, the simulated spicule expands with height, which also acts to decrease the rise of the vertical velocity with temperature (panel (B) in Figure 6).

The reason for the discrepancy between the actual vertical velocities and the Doppler shifts can be appreciated in the top panels of Figure 15. This figure shows the emission as a function of temperature and vertical velocity for the various lines. For the transition region lines (C IV $195 \AA$, N v $1238 \AA$, O VI $195 \AA$ ), the emission is concentrated in two velocity regions, one around $0 \mathrm{~km} \mathrm{~s}^{-1}$ and the other around $60 \mathrm{~km} \mathrm{~s}^{-1}$. The Doppler velocity of these profiles, using a single Gaussian approximation, depends on the upward velocity and the contribution of the main emission and also the location and contribution of the second emission region. These two concentrations of emission are coming from the surrounding of the spicule $\left(0 \mathrm{~km} \mathrm{~s}^{-1}\right)$ and the upper part of the spicule $\left(60 \mathrm{~km} \mathrm{~s}^{-1}\right)$. In contrast, hotter (coronal) lines have a single region of emission spread over a large velocity range and the integrated emissivity decreases with increasing vertical velocity. The three main contributions to the coronal line emission are 

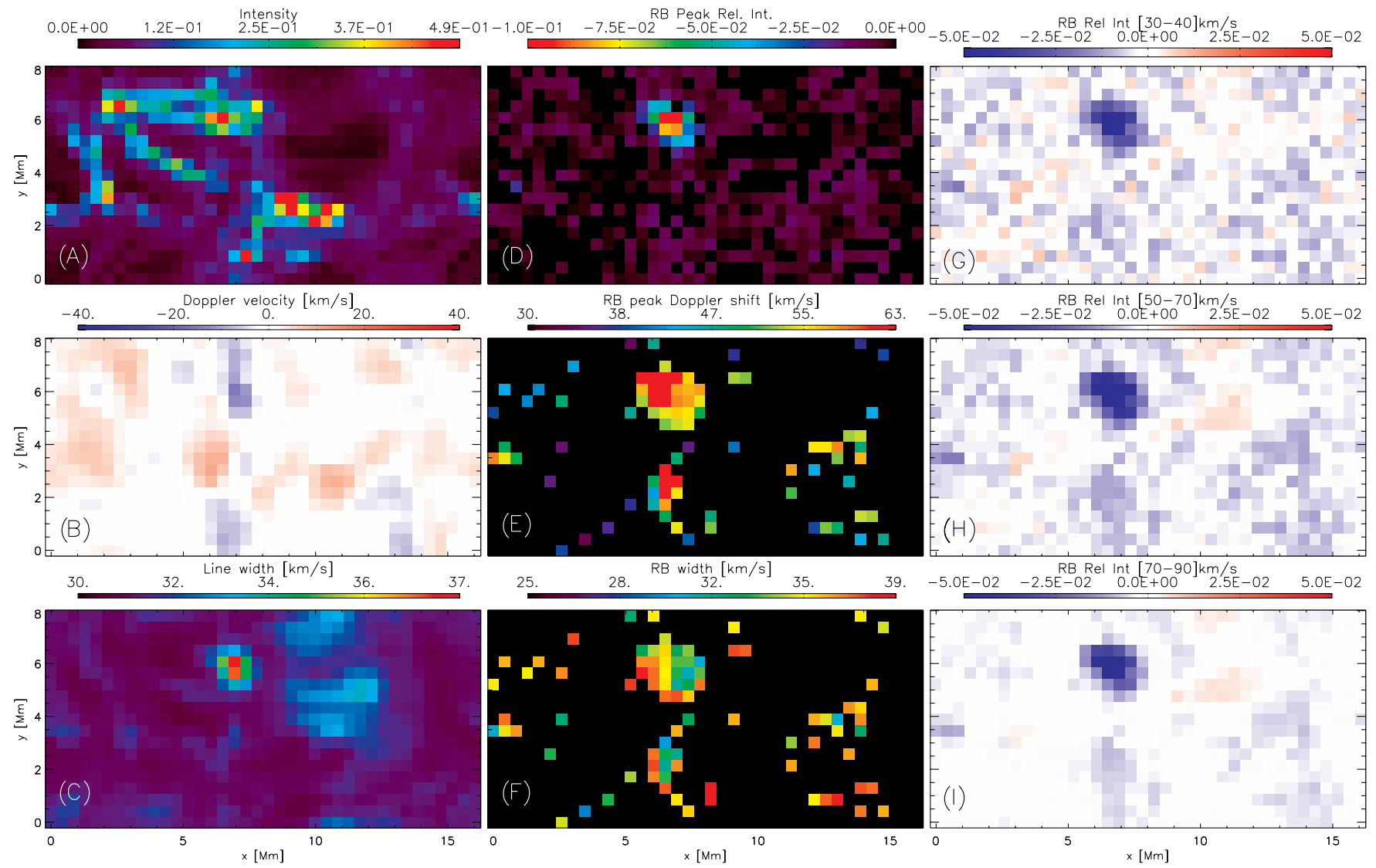

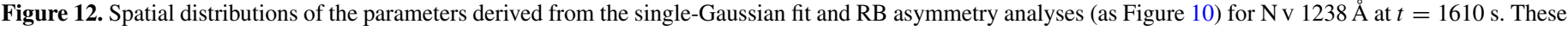
profiles are calculated taking into account Hinode spatial resolution and instrumental broadening of spectral lines.

(A color version of this figure is available in the online journal.)

the spicule, the hot loop associated with the spicule, and the hot corona overlying the simulated domain. Because of the large scale height of coronal lines, we sample a wide range of velocities and emissivities. Figure 15 clearly illustrates that using only the Doppler shift information of the line does not necessarily provide accurate information about the real velocity of the plasma at the different formation temperatures. In fact, using only the Doppler shift can lead to the wrong conclusion: the Doppler velocity increases significantly for ions with the formation temperature but the vertical velocity of the plasma does not increase as much with temperature (Figure 15). Therefore, it is crucial to have a complete study of the line profile and include also the RB asymmetries (bottom panel in Figure 14). The coronal lines show stronger Doppler velocities because their emission does not have as much contribution from low velocities as the transition region lines and the strongest emission is located at higher velocities (Martínez-Sykora et al. 2011a). As a result of this, the blueward RB asymmetries of the coronal lines are shifted to lower velocities compared with the transition region lines. We note that this conclusion is valid for the current simulation, which does not have much overlying coronal emission. On the Sun, the impact of the LOS superposition on Doppler shifts and line asymmetries will critically depend on the overlying background emission.

Inside the spicule, the line width of the spectral lines is larger for the various lines shown in the middle panel of Figure 14 than in the other selected regions. The line width also increases with increasing formation temperature up to $1.2 \times 10^{6} \mathrm{~K}$. This linear increase becomes smaller around at $\log (T)=5.8$ because the hotter lines are emitted by non-lithium-like ions, which have a different temperature dependence of the contribution function $\left(G\left(T, n_{e}\right)\right)$. For instance, lithium-like ions (C IV $195 \AA$, N v $1238 \AA$, O vi $195 \AA$, and Si vir $275 \AA$ ) show a significant high-temperature tail of the $G\left(T, n_{e}\right)$ compared to non-lithiumlike ions, such as Fe IX-XII (see Figure 13 in Martínez-Sykora et al. 2011a). As a result of this, lithium-like ions show contributions coming from plasma with a wider range of temperatures than non-lithium-like ions; and the line width will be larger for the lithium-like ions as a result of the LOS integration. Such behavior can be seen also in other regions such as the loop region or the transition region footpoints.

The time evolution of the properties of a representative transition region spectral line ( $\mathrm{N} \mathrm{v} 1238 \AA$ in black), a representative coronal line (Fe x $184 \AA$ in red), and an intermediate line (Si VII $275 \AA$ in green) are shown in Figure 16 . In order to calculate the velocity (bottom-middle panel) and width (top-right panel) of the RB asymmetry profile, we limit this to RB asymmetries larger than $0.5 \%$ of the peak intensity. Before the spicule emerges all the lines show blue Doppler shift (positive top-left panel), which is larger for ions with higher formation temperature. At the time the spicule appears $(t=1470 \mathrm{~s})$, the Doppler shift increases with time for all lines, and the increase is largest for coronal lines. However, it is interesting that the duration of high Doppler shift is shorter for transition region lines (C IV $195 \AA, N_{\text {V }} 1238 \AA$, and O VI $195 \AA$ ) than for the coronal lines (Fe IX $188 \AA$, Fe x $184 \AA$, and Fe XIV $274 \AA$ ). Similar behavior is observed also in the other properties of the spectral lines, such as the line width (bottom-left panel), the RB intensity (middle 

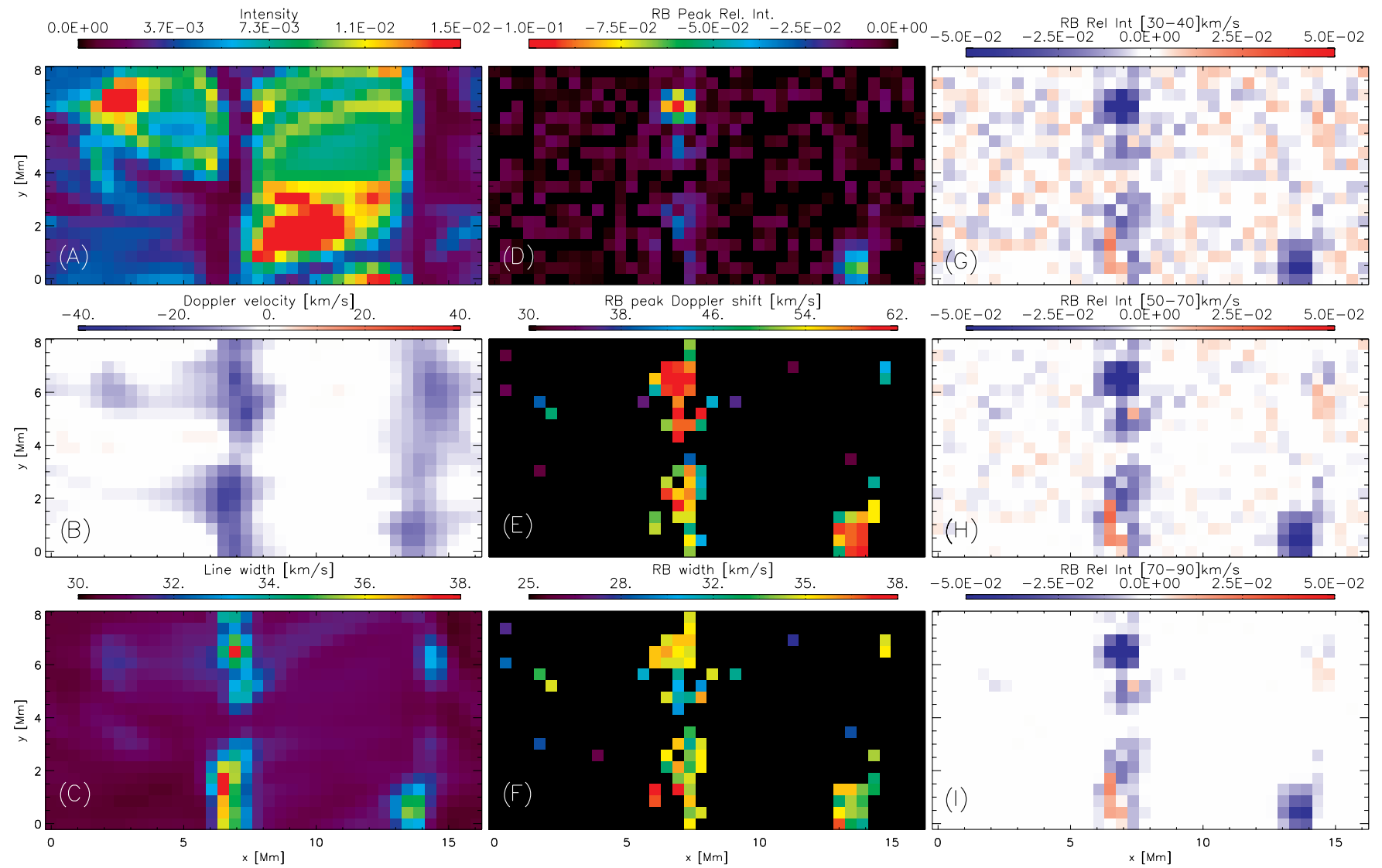

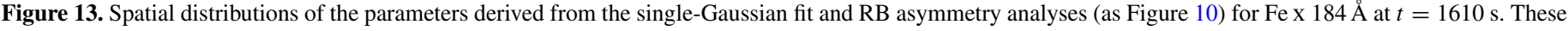
profiles are calculated taking into account Hinode spatial resolution and instrumental broadening of spectral lines.

(A color version of this figure is available in the online journal.)

top), the velocity of the RB peak asymmetry (bottom-middle panel), the width of the RB asymmetry profile (top-right panel), and the RB asymmetry around 50-70 $\mathrm{km} \mathrm{s}^{-1}$ (bottom-right panel). All of these properties increase at the same time as the spicule evolves but this increase is shorter-lived for the transition region lines than for the coronal lines. This suggests that trying to estimate the lifetime of the coronal counterparts of spicules based on transition region diagnostics might lead to misleading results. In particular, it suggest that the results of Klimchuk (2012), who cast doubt on the impact of spicules on the corona, likely significantly underestimate the real lifetime of the coronal counterpart of spicules.

During the early phase of the spicule, most of the spicular plasma is at chromospheric and transition region temperatures and has a wide range of velocities because of the complex acceleration mechanism. This leads to strong Doppler shifts, line widths, and asymmetries. The coronal emission "riding" on top of the spicule will also show a similar increase of the spectral line parameters. Later in time, the spicule is heated and the transition region lines show only the footpoints of the spicule, whereas the coronal emission occurs throughout the spicule. As result of this, the transition region lines show the emission of a limited volume (height) where the velocities are all very similar and small as shown in the bottom panels of Figure 15. Therefore, the LOS integration is limited to a thinner region and as a consequence, the Doppler velocity, the line width, and the RB asymmetries no longer show an increase in time for transition region lines. In contrast, the coronal lines maintain the RB asymmetries as a result of the dynamics of the spicule (upflows) and remain even after the chromospheric material is heated. Note that if time dependent ionization of the various ions is taken into account, the diagnostics may change (Hansteen 1993; Bradshaw 2009; Judge et al. 2012; Olluri et al. 2013).

It is also interesting that the transition region lines show the strongest asymmetries, and largest shift and width of the RB peak asymmetry. As mentioned above, for transition region lines, the emission comes from two regions, one in the footpoints of the spicule and the second one from the top of the spicule. As a result of this, the asymmetries are larger than in coronal lines. We want to highlight again the importance of studying the Doppler shifts together with the asymmetries of the spectral lines. Failure to do so can easily lead to incorrect conclusions.

It is crucial to mention that in the synthetic observables we do not have very strong background emission because of the small size of the computational domain. The background emission impacts the properties of the profiles such as Doppler shifts, and asymmetries (Martínez-Sykora et al. 2011a).

4.2.2.2. At the limb. At the limb, the Doppler velocity map of synthetic chromospheric lines such as $\mathrm{H} \alpha$ shows red- and blueshift at each side of the spicule (see Figure 17) as if the plasma is rotating at the same time that it is ejected into the corona. The Doppler velocities observed at the limb are of the order on $\pm 10 \mathrm{~km} \mathrm{~s}^{-1}$. Such torsional motions have recently also been observed by De Pontieu et al. (2012) in $\mathrm{Ca}$ II H. The Doppler velocities are larger in the observations than the velocities observed in the synthetic profiles. However, the difference seems similar to the discrepancies between the various properties of the synthetic RBE (e.g., Doppler velocities 

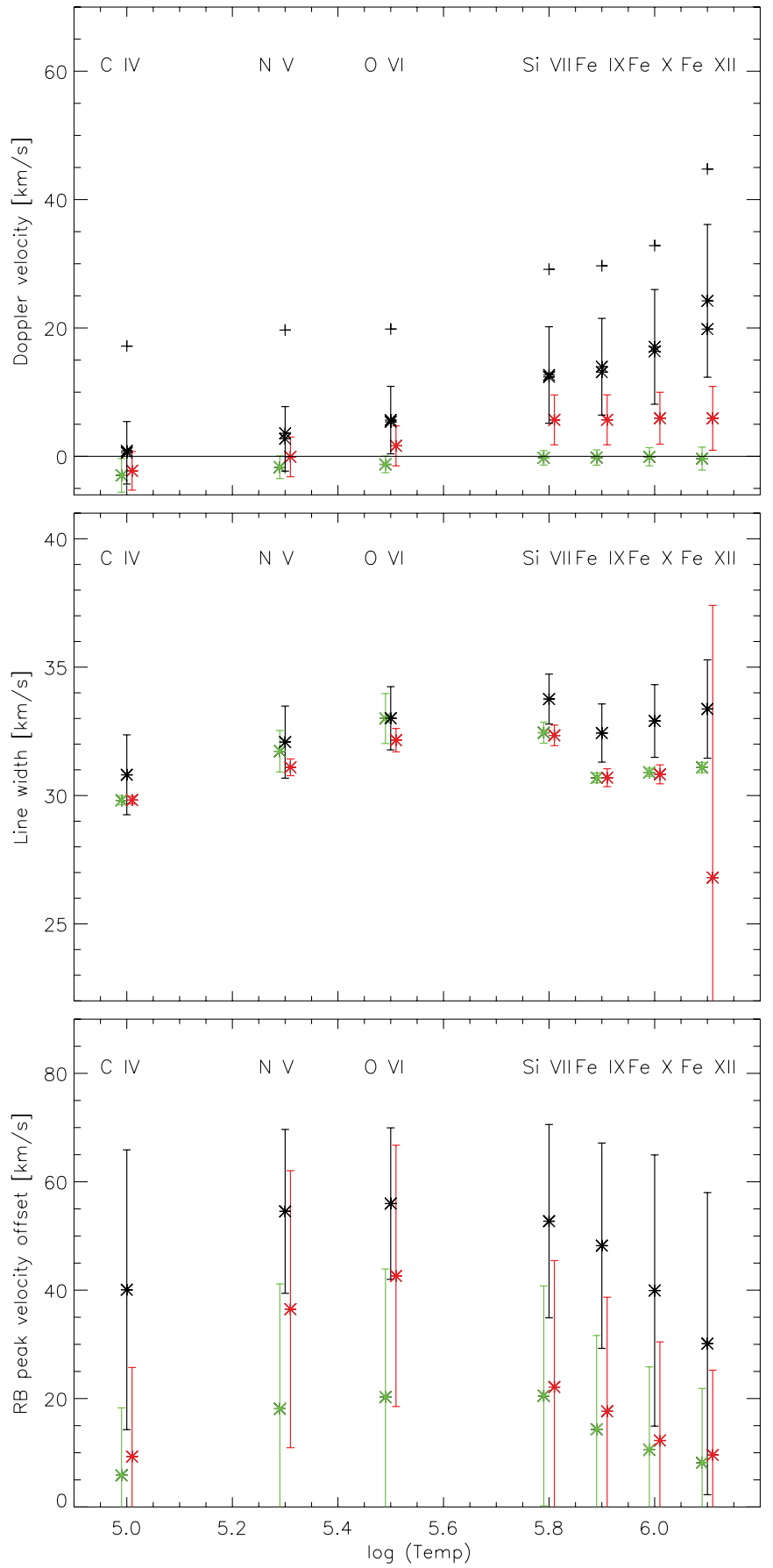

Figure 14. Mean (asterisks) and standard deviation (error bars) of the Doppler shift (top panel), line width (middle panel), and the Doppler velocity of the RB peak asymmetry (bottom panel) at time $t=1600 \mathrm{~s}$ for different lines shown as a function of the maximum formation temperature of each ion. The profiles have been degraded to the spatial resolution of Hinode and instrumental broadening of the spectral line profile. These values are calculated within three different regions: the loop footpoints (the region delimited with the red square shown in panel (B) in Figure 10), the loop regions (the region delimited with the green square in panel (B) in Figure 10), the spicule (the region delimited with the black square) is shown in red, green, and black, respectively. The + symbol in the top panel refers to the maximum Doppler shift. The Doppler velocity of the RB peak asymmetry is shown only when the RB asymmetry is more than $0.5 \%$ of the total local intensity.

(A color version of this figure is available in the online journal.)

in the disk view) in comparison with the observations. Once again, these results suggest similarities with the observations but also indicate that this simulated spicule is not as violent as the type II spicules on the Sun.
Another main difference between the observations and the synthetic profile is that in the observations (De Pontieu et al. 2012) the spatial variation of the spectral profile (bottom panel of Figure 17) does not show two treads. Such threads are seen in the synthetic case. These threads appear as a consequence of the variation of density within the spicule. The multi-threaded nature of the simulated spicule is, however, compatible with suggestions of such structuring in observations (Suematsu et al. 2008).

This Doppler shift pattern at the limb is observed also in transition region and coronal lines as shown in Figure 18. This effect can be seen in any LOS direction at the limb, i.e., when integrating along the $x$-axis instead of the $y$-axis the red-blue Doppler shift structure is still visible. This Doppler shift is not uniform or constant in space as a function of the formation temperature of the various lines, and also the evolution seems to differ between the various lines. The transition region lines show such Doppler shift structure only when the chromospheric material is injected into the corona, before it is heated. The Doppler shifts in the transition region lines are larger than in the coronal lines but smaller than in the chromospheric lines. In addition, for Fe IX-XII this Doppler shift structure seems to last for a shorter time. For Fe XIV $274 \AA$, the red-blue Doppler shift appears later because the plasma needs to be heated to high temperatures. It also lasts longer than for the other cooler coronal lines.

The Doppler shifts in the various limb views suggest the spicule is rotating. We confirm this by studying the vorticity in the simulations as shown in Figure 1 with the velocity streamlines, but what causes it to rotate in the chromosphere and corona? One can derive a conservation equation of the vorticity by taking the curl of the MHD equations:

$$
\begin{aligned}
& \frac{D \omega}{D t}=(\omega \cdot \nabla) \mathbf{u}-\omega(\nabla \mathbf{u})+\frac{1}{\rho^{2}} \nabla \rho \times \nabla p \\
& +\frac{1}{\rho^{2}} \nabla \rho \times\left[\nabla p_{\text {mag }}-\frac{1}{4 \pi}(\mathbf{B} \cdot \nabla) \mathbf{B}\right] \\
& +\frac{1}{4 \pi \rho} \nabla \times(\mathbf{B} \cdot \nabla) \mathbf{B},
\end{aligned}
$$

where $\omega=\nabla \times \mathbf{u}, p, \rho, \mathbf{B}$, and $p_{\text {mag }}=\left|B^{2}\right| /(4 \pi)$ are the vorticity, gas pressure, density, magnetic field, and the magnetic pressure. The terms in the right side of the equation are the tilting, stretching, baroclinic, magneto baroclinic terms, and magnetic tension (Shelyag et al. 2011; Steiner \& Rezaei 2012). Figure 19 shows in an early stage of the spicule ejection the vertical cut of the vorticity, the velocity perpendicular to the plane $\left(u_{y}\right)$, and the two most important terms on the right side of Equation (2) in the chromosphere and corona, i.e., the magneto baroclinic term and the magnetic tension. As a result of the compression at the footpoint of the spicule due to the magnetic field tension, the magneto baroclinic term generates a vorticity not only inside the chromosphere but also all the way up into the corona. This vorticity is generated in the chromosphere at the footpoint of the spicule, and it is the result of the compression. The magnetic baroclinic term is important because the density decreases along the $y$-axis and this density gradient is perpendicular to the gradient of magnetic pressure that is perpendicular to the magnetic field. In addition, the gradient of magnetic pressure perpendicular to the field lines is large along the $x$-axis. This is a consequence of the plasma being compressed, resulting in the generation of vertical vorticity. 

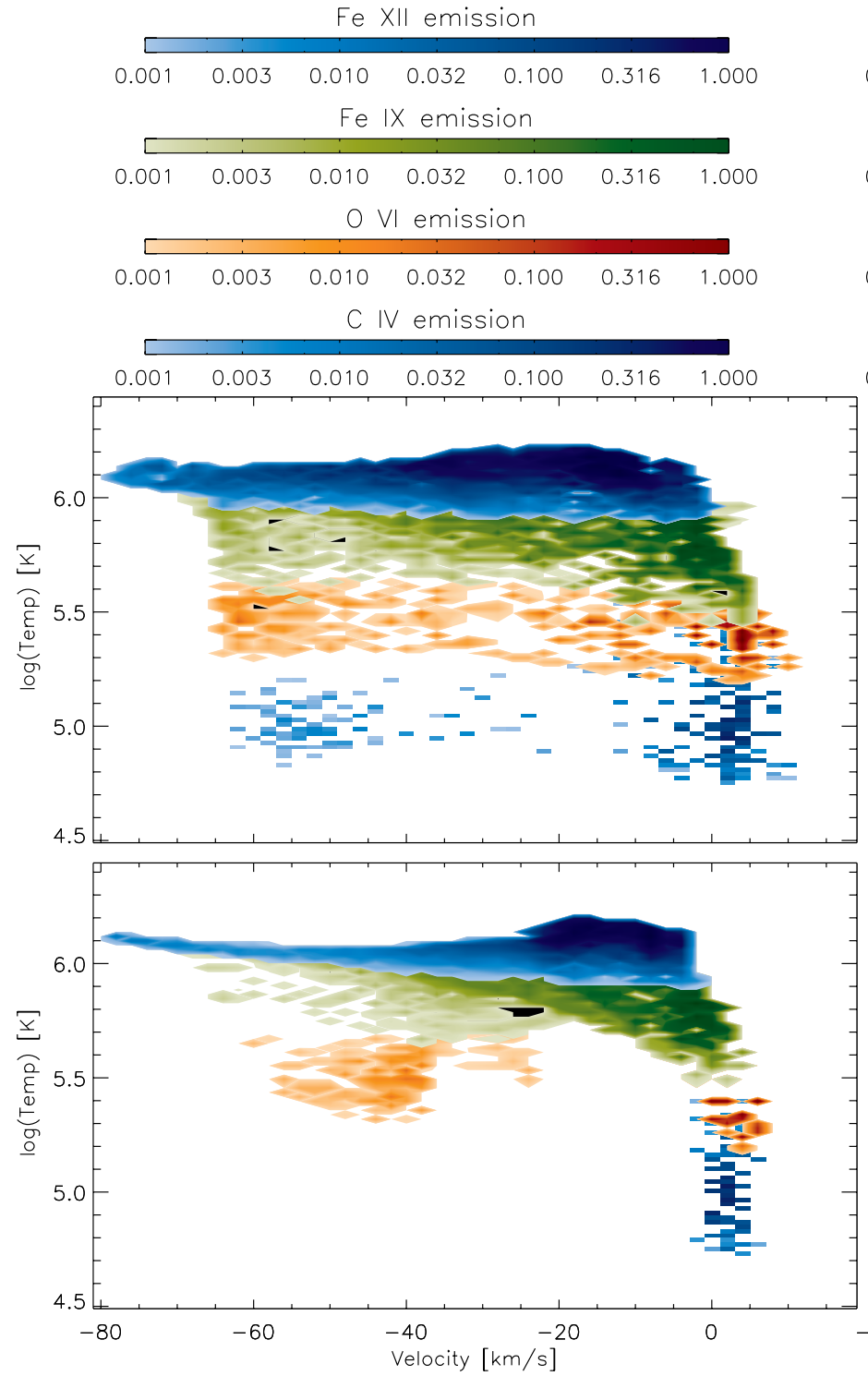

\begin{tabular}{|c|c|c|c|c|c|c|}
\hline 0.001 & 0.003 & 0.010 & 0.032 & 0.100 & 0.316 & 1.000 \\
\hline \multicolumn{7}{|c|}{$\mathrm{Fe} X$ emission } \\
\hline 0.001 & 0.003 & 0.010 & 0.032 & 0.100 & 0.316 & 1.000 \\
\hline \multicolumn{7}{|c|}{ Si VII emission } \\
\hline 0.001 & 0.003 & 0.010 & 0.032 & 0.100 & 0.316 & 1.000 \\
\hline
\end{tabular}
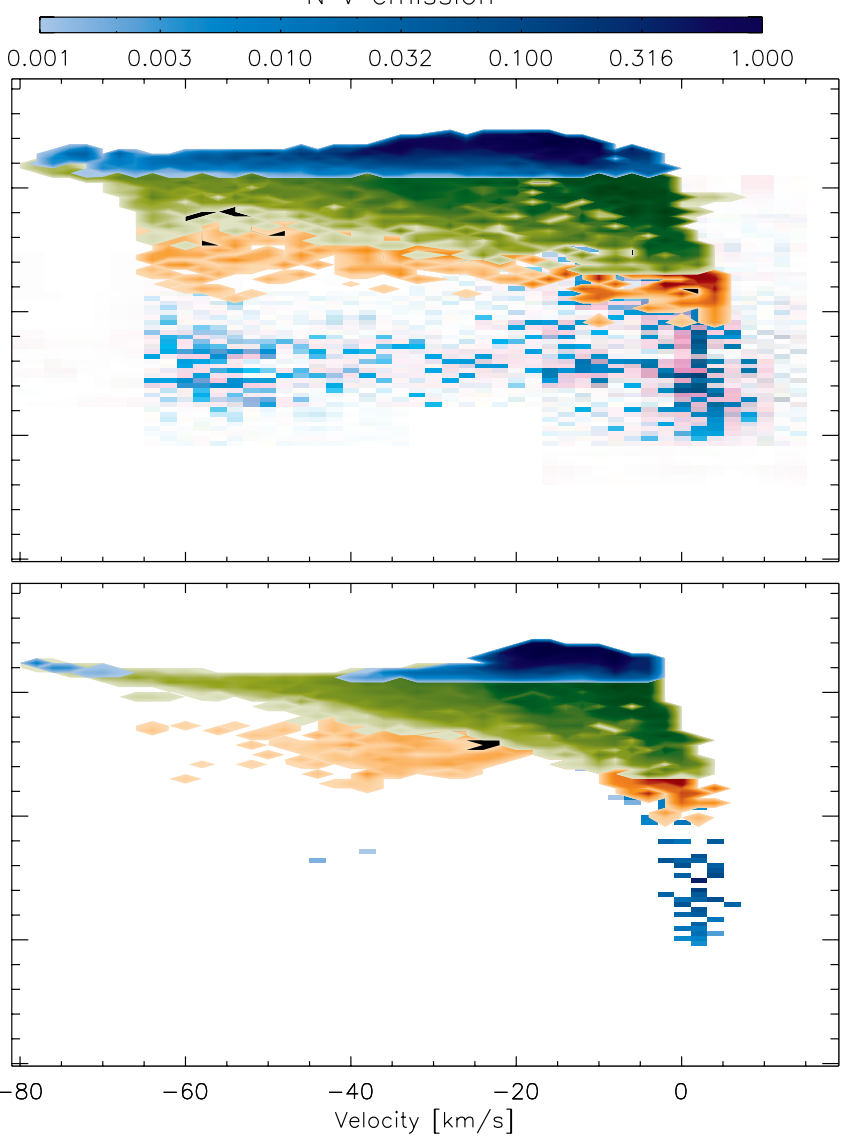

Figure 15. Emission as a function of temperature and vertical velocity in the region of plasma confined within one Hinode pixel centered at $x, y=[5,6] \mathrm{Mm}$ at $t=1600 \mathrm{~s}$ (top panels) and at $t=1680 \mathrm{~s}$ (bottom panels). It is shown for different lines, which follow the different color schemes displayed on top. Fe XII $195 \AA \AA$ and C IV $195 \AA$ in the left panels and Fe XIV $274 \AA$ and N v $1238 \AA$ in the right panels share the same color scheme, but the emission coming from C IV $195 \AA$ and Nv $1238 \AA$ is centered around $\log (T) \sim 5.0$, and Fe XII $195 \AA$ and Fe XIV $274 \AA$ are centered above $\log (T) \sim 6.1$.

(A color version of this figure is available in the online journal.)

\subsection{Comparison with Observations}

De Pontieu et al. (2011) used SDO/AIA observations to show the impact of type II spicules into the corona using SDO/AIA. Here we use the same data set to see whether we can recognize some similar features in the simulated spicule. Figure 20 shows a time series of an RBE visible as a dark feature in $\mathrm{H} \alpha$ (-868 $\mathrm{m} \AA$ ) (top row). Along the RBE the second and third rows of the figure show a brightening at transition region $(304 \AA$ channel) and coronal (211 A channel) temperatures (De Pontieu et al. 2011). Note that the features are not overlapping exactly in space and time. This property agrees with the simulation. In the simulation, the emission from different lines shows different structures and do not overlap because the heating is not uniformly located and the various temperature structures in the spicule and surroundings are complex. As mentioned before, the fact that the brightenings do not share the exact location and instant implies that the $304 \AA$ channel most likely shows transition region temperatures ( $\mathrm{He}$ II $304 \AA$ ) and the $211 \AA$ channel shows coronal temperatures (Fe XIV $211 \AA$ ).

There are two major features that catch our attention in the time evolution of the brightening in the AIA channels shown in Figure 20. One of these is hardly seen in the modeled spicule. This brightening is following the spicule in the SDO/AIA channels and disappears almost as quickly as the spicule fades. This was observed by De Pontieu et al. (2011). This brightening is small in the simulation in comparison with the observations. This may be a consequence of the simulated spicule being not as violent as the observed spicules. The other feature seems to be more evident in the simulations than in the observations but still observed here in the various SDO/AIA channels. This is the brightening at the footpoint of the RBE, which lasts for a few hundred seconds, and seems to increase in time. This may indicate that the heating mechanism persists longer than the lifetime of the RBE. Other examples of this type of observations are shown in Figure 21, which shows 

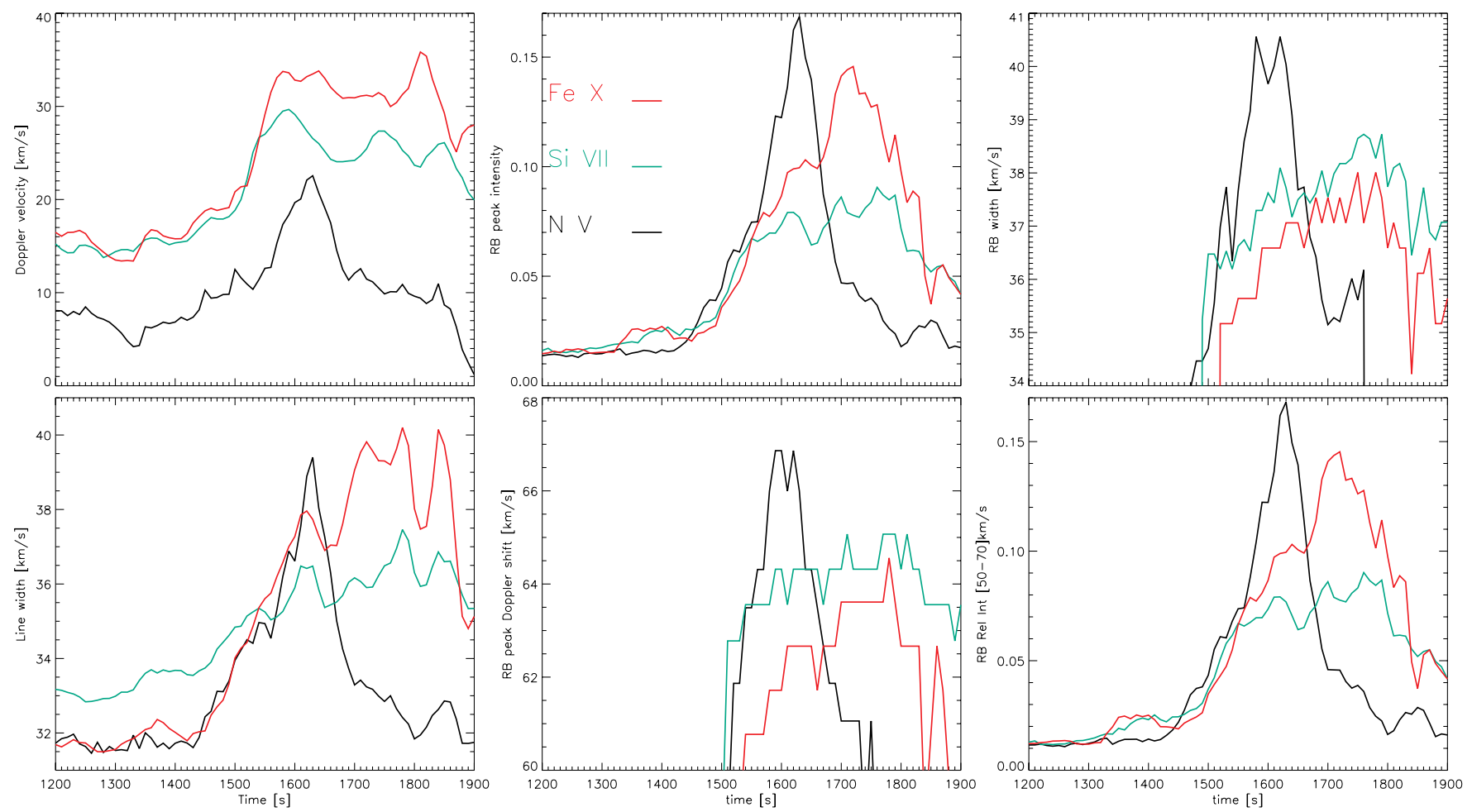

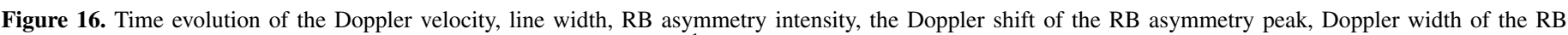

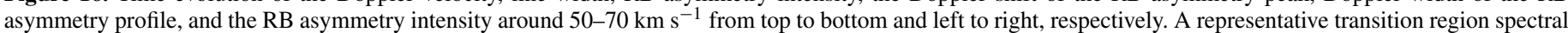

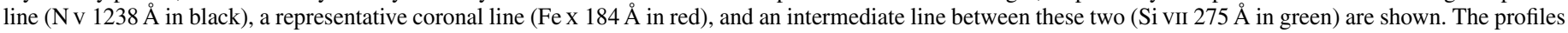

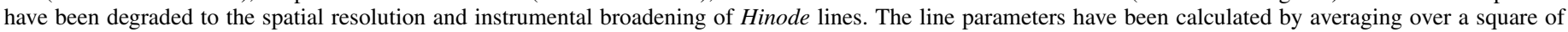
$3 \times 3$ pixels where the spicule is located.

(A color version of this figure is available in the online journal.)

time-space plots where these two features in the time evolution can be seen in one or more of the SDO/AIA channels. Note that these data are pushed to the limit of their spatial and temporal resolution and signal to noise to enhance the brightening. We find that in the AIA observations it is more common to see an increase of brightening in the $304 \AA$ channel than in the coronal emission. In contrast, in the simulation we see the opposite. This discrepancy could be explained by two issues. First, the modeled magnetic field configuration is different from that on the Sun. In the simulation the magnetic field is confined to a small domain and the magnetic field lines that produce this strong coronal emission close within the computational domain. Therefore, the heat and high temperatures are confined on these lines, so thermal conduction does not strongly dilute the heat input. This may lead to a modeled coronal emission that is brighter than on the Sun. Second, the formation of He II $304 \AA$ is not well understood (Feldman et al. 2010), which likely leads to discrepancies between our calculations and the observations.

Figure 22 shows SUMER observations of Nv $1238 \AA$ of a quiet Sun and network region. The intensity of the line is slowly changing in the bright network region over the course of the one hour long time series. The eight RB panels show (once again), in the magnetized network, redward asymmetry for velocities less than $50 \mathrm{~km} \mathrm{~s}^{-1}$ shifting rapidly to blueward asymmetries for velocities in excess of $50 \mathrm{~km} \mathrm{~s}^{-1}$. In the internetwork regions very little asymmetry is found for these velocities.

Further analysis of the Nv $1238 \AA$ time series shows that the characteristic timescale on which these upflow events seem to recur is of order $3-15$ minutes. This is illustrated on the right side of Figure 22, which shows, as a function of time and space, the episodic nature of the RB asymmetry of the $\mathrm{N} \mathrm{v}$ line (summed from 58 to $111 \mathrm{~km} \mathrm{~s}^{-1}$ ). This is confirmed by a wavelet analysis (using the Morlet wavelet) of the RB time series: the resulting wavelet power spectrum (bottom right panel) shows significant (at 95\% significance level) power throughout the time series at periods of order 3-5 minutes, as well as some at 15 minutes. From the models, we can not undertake this type of study since we were not able to simulate the recurrence of these events. The middle panel shows the RB time series at $y=-22^{\prime \prime}$ with clear peaks of blueward asymmetry occurring, and typical events lasting of order 1-3 minutes. It is also interesting to see that the strong intensity lasts for more than 5 minutes for the event at $y=-22^{\prime \prime}$, i.e., a few minutes more than the RB asymmetry signal. This lifetime of the strong intensity signal seems to agree with the simulations and the $S D O /$ AIA results as mentioned above.

Figure 23 shows EIS observations of several coronal lines of an active region. From this data set, Tian et al. (2012) studied the recurrence of these signals. Here we are interested in the duration of a particular event. Around $t=20$ minutes there is an event in the hotter lines that lasts for 5 minutes in intensity and Doppler shift, and the RB asymmetry and line width starts later and seems to last shorter (3-4 minutes). Similarly, around $t=15$ minutes for the cooler lines (Si VII $275 \AA$, Fe X/XII $184 \AA$ ), the intensity Doppler shift and RB asymmetries last for more than 3 minutes. Note the large difference of the lifetime of these events in the coronal lines ( $\sim 5$ minutes) and the transition region lines (1-3 minutes).

The lifetime of the RB asymmetries in the transition region lines as shown with SUMER in Figure 22 is shorter 

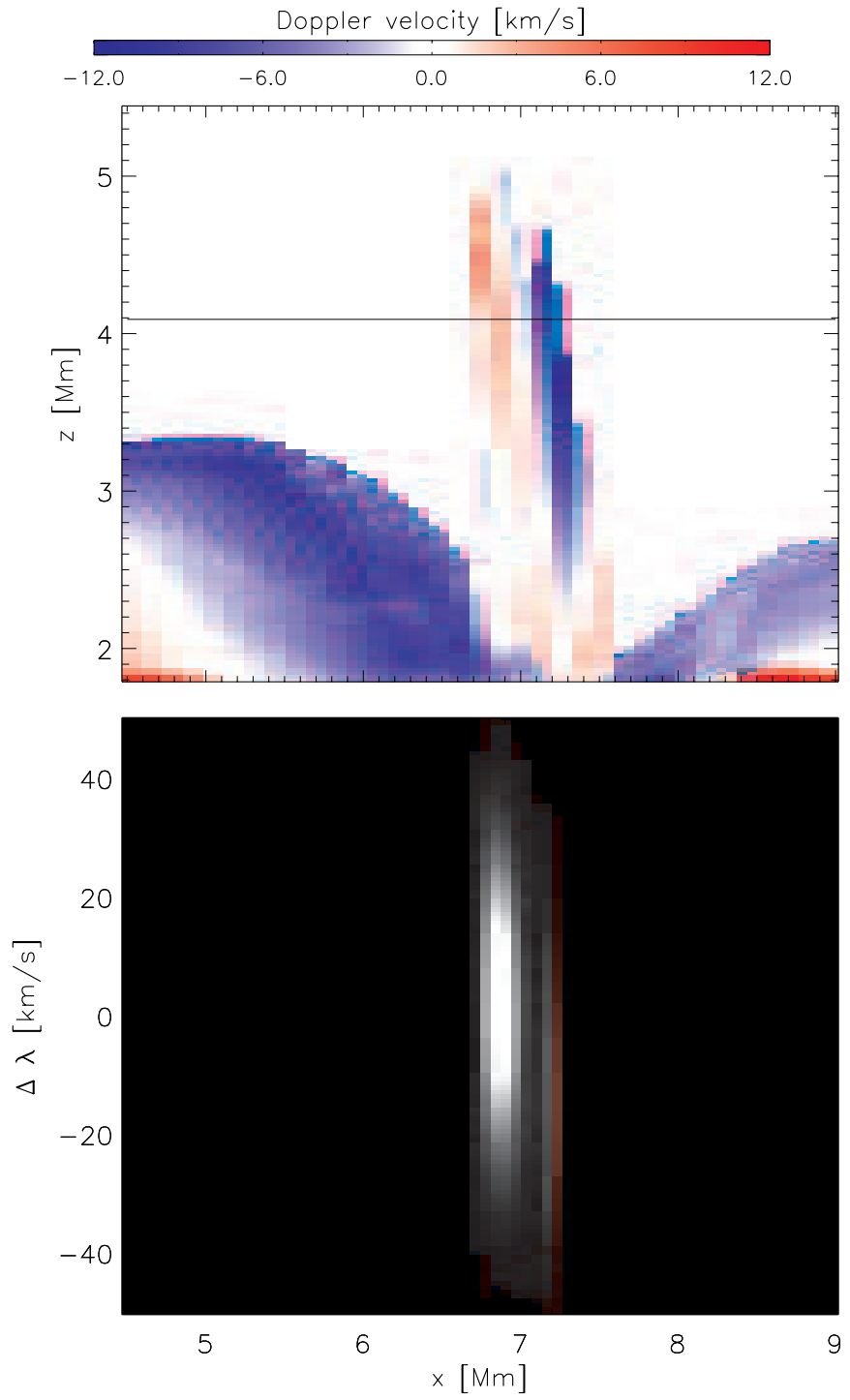

Figure 17. Off-limb view of large torsional motion in the simulated spicule in $\mathrm{H} \alpha$. Doppler shift map at the limb (i.e., the $x z$-view) is shown in the top panel and the spatial variation of the $\mathrm{H} \alpha$ profile at $z=4.1 \mathrm{Mm}$ is shown in the bottom panel.

(A color version of this figure is available in the online journal.)

(1-3 minutes) than the lifetime of the RB asymmetries and Doppler shifts of the coronal lines (Figure 23). This is very similar to what we see in the simulations. From the model, the transition region lines show large $\mathrm{RB}$ asymmetries for a lifetime of roughly 2-3 minutes, but the coronal lines shows large RB asymmetries for $\sim 5$ minutes as described Section 4.2.2.1. Unfortunately, we do not have access to RB asymmetry maps of an identical region from the observations for both temperature regimes (transition region and coronal lines). In addition, we do not have simultaneous $\mathrm{H} \alpha$ observations to link the events with RBEs. Therefore further observational studies are needed.

\section{DISCUSSION AND CONCLUSIONS}

We have studied the thermodynamics of a plausible candidate for a type II spicule model using chromospheric, transition region, and coronal synthetic observables and compared them with observations. The driver of this candidate spicule has been studied by Martínez-Sykora et al. (2011), who found that it evolved naturally as a consequence of the magnetic field dynamics of the model (which include flux emergence). In short, chromospheric material is ejected into the corona as plasma is squeezed horizontally by a strong Lorentz force. The pressure increases and thus deflects the plasma and forces it to flow vertically along the magnetic field, most rapidly toward the low densities of the corona. Several synthetic observables reproduce the observations. However, mostly in the chromosphere, some synthetic properties differ from the observations.

The chromospheric diagnostics seem harder to reconcile with the observations than coronal diagnostics for obvious reasons: the chromosphere is a transition between the photosphere and corona where a mixture of various physical processes play a role, such as non-LTE, non-equilibrium ionization, ion-neutral interaction effects, etc. In addition, calculating the synthetic observables is more complex for chromospheric lines than for coronal lines. In addition to this complexity, it is also clear that the simulated spicule is not very violent and shows chromospheric densities that seem to be too low by at least an order of magnitude. All of these issues likely help explain why the simulated spicule does not show a clear RBE signal: because of the low hydrogen density, the opacity is too low above $z=2 \mathrm{Mm}$ for the unclear absorbing component in the wings of the synthetic profiles can be detected. Such a component is observed in RBEs on the Sun. Even though we find large differences between the observed and synthetic profiles, especially as seen on the disk, it is important to note that one can deduce what is missing and/or could help to mimic the observables by studying the differences in detail. The low density indicates that not enough chromospheric material is ejected into the corona and/or, as a result of the simplified magnetic field configuration, the spicule expands too fast in the corona due to the field expansion. We note that, despite discrepancies between synthetic and real chromospheric observables, the physical properties such as the plasma being heated and accelerated along the spicule does seem to correspond with what is observed on the Sun.

The off-limb observables seem to show better agreement than the on-disk observables. The synthetic Ca II $8542 \AA$ and $\mathrm{H} \alpha$ emissions show structures and evolution that are (in part) similar to the observations. Even though apparent upward velocities seem too low, the chromospheric signal of the synthetic spicule fades in a few seconds (similar to observations). In the simulations this occurs as a result of being heated mainly by Joule heating, and to a lesser extent by thermal conduction and advection. However, the synthetic Ca II $8542 \AA$ and $\mathrm{H} \alpha$ emission are fainter than the observed ones, perhaps because the spicule does not eject enough chromospheric material. It is interesting that we "observe" torsional behavior in the spicule that is similar to the observations (De Pontieu et al. 2012). This rotation is seen not only in chromospheric lines but also in transition region lines and to a lesser extent in the coronal lines. This rotation is generated in the chromosphere as a result of the compression, which leads to a high magneto baroclinic term. It is interesting to note that all the chromospheric dynamics of the spicule are at the low end of the observed range, i.e., both upflows and rotation velocities are lower compared with observations by the same relative factor.

The spicule shows emission in several transition region and coronal EUV lines (He II $304 \AA$, Fe IX-XIV, etc.). The transition region lines show emission at the limb following a path that is similar to a parabolic profile and coronal lines show a wave like propagation into the corona $\left(\sim 100 \mathrm{~km} \mathrm{~s}^{-1}\right)$. The former type of evolution is a result of various heating contributions, and it does not show downflows. The latter type of evolution is 


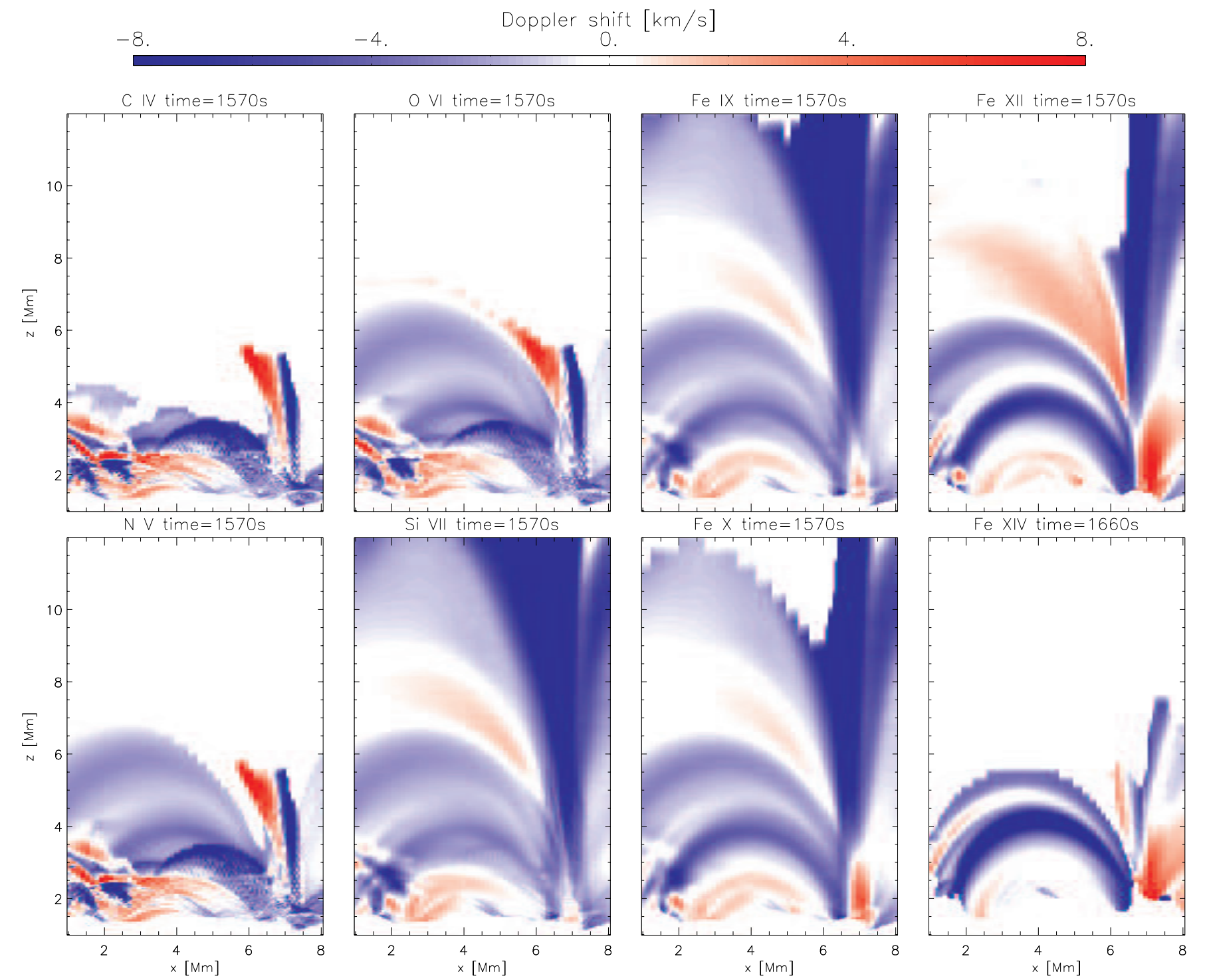

Figure 18. Doppler shift maps at the limb (i.e., the $x z$-view) for different lines. Note that the spicule shows in the left side a redshift and in the right side a blueshift.

(A color version of this figure is available in the online journal.)

a combination of waves, flows, and a thermal conduction front. These evolutions seem in agreement with the observations (De Pontieu et al. 2011). On the disk, two different brightenings have been observed in transition and coronal lines, one with the ejection of the spicule, and another one that increases slowly with time over a range of a few hundred seconds. Some observational examples seems to have similar properties, but the brightenings from the observations are weaker than the synthetic ones. This difference may be a result of the magnetic field configuration, the computational domain is small and the heating on the Sun can be expanded to larger distances along the magnetic field lines than in our simulation.

The transition region and coronal lines show not only blue Doppler shifts but also strong asymmetry and an increase of the line width in the spicule. In addition, we find a different time evolution of the properties of the profiles for transition region lines and coronal lines. The increase in Doppler shift, line width, and asymmetry lasts significantly longer in coronal lines. We show some observational examples of the duration of the Doppler shifts and RB asymmetries of transition region and coronal lines where the synthetic results seem to agree with the observations. However, further studies are needed because we were not able to compare transition region lines with coronal lines in the same region and time on the Sun. In addition, in the observations we can not link the RB asymmetries events with RBEs because we did not have simultaneous $\mathrm{H} \alpha$ observations. We also want to point out that it is crucial to combine the information of the Doppler shift and the asymmetry of the line in order to correctly determine the plasma velocities. Using only Doppler shift or only asymmetries is likely to lead to incorrect conclusions about plasma velocities. We also take into account the effects of the spectral broadening and spatial resolution into these results. Some of these lines may be impacted by the nonequilibrium ionization (Judge et al. 2012).

Several processes still need to be taken into account that will likely impact the synthetic diagnostics. At a minimum we need to include ion-neutral coupling effects, and non-equilibrium ionization. The latter plays a role for the energy properties of the chromospheric plasma (Leenaarts et al. 2012), but also in some transition region EUV line diagnostics (Olluri et al. 2013). It is 

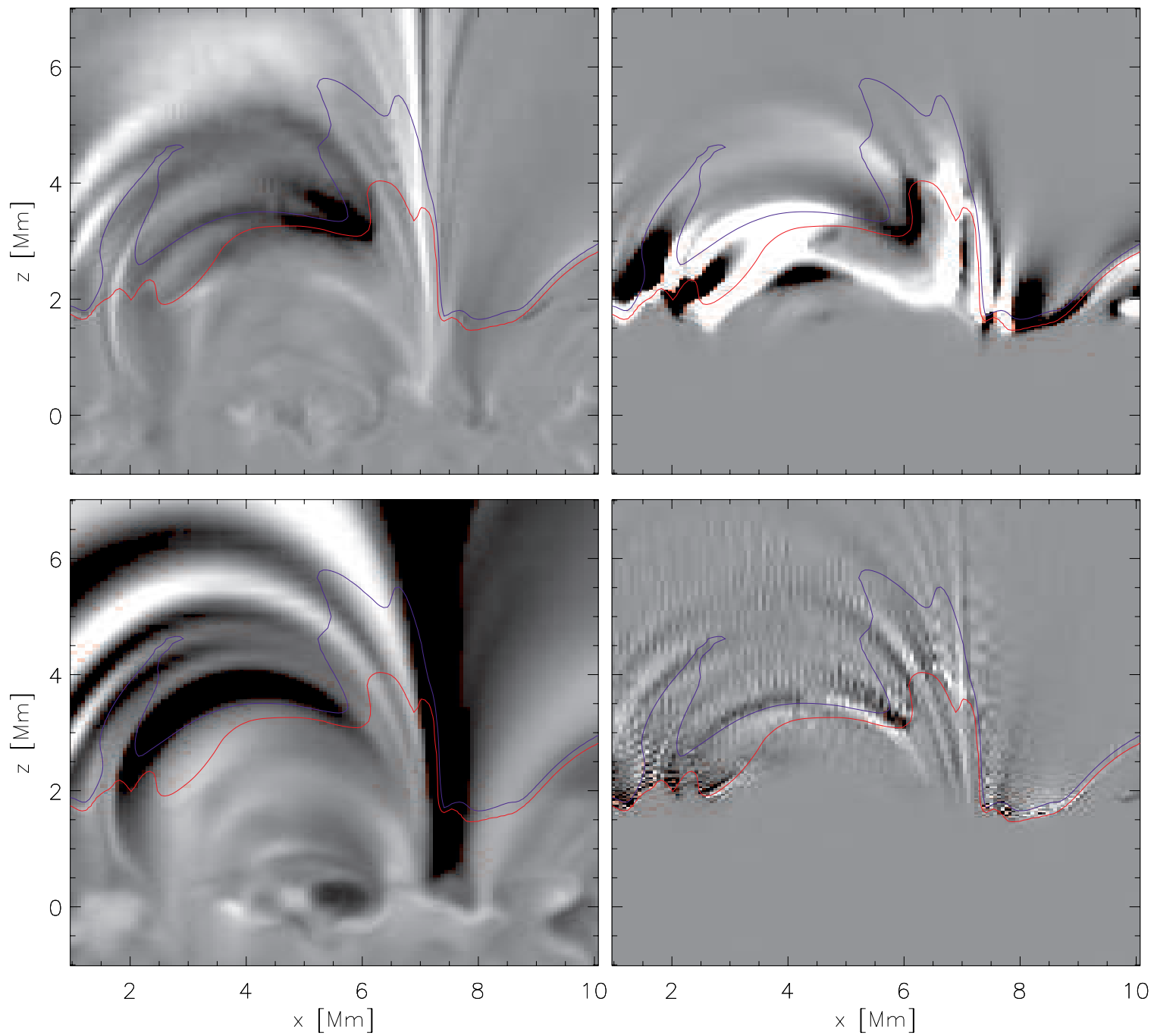

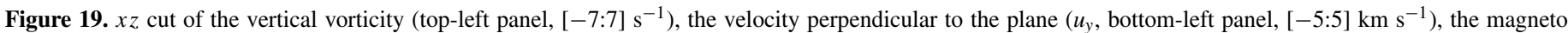

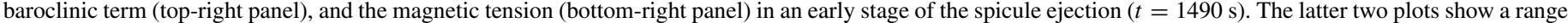
covering $[-2: 2] \mathrm{s}^{-2}$. The temperature contour at $2 \times 10^{5}$ and $5.5 \times 10^{5} \mathrm{~K}$ are overlaid with red and blue colors, respectively.

(A color version of this figure is available in the online journal.)

also crucial to increase the spatial resolution of the simulations and keep in mind that the Joule heating in the model is dependent on numerical diffusivities and dissipation and that heating on the Sun behaves differently.

Many properties of type II spicules, both from the observations and the model presented here, indicate that it cannot be modeled properly in 1D models. For instance, from the observational point of view, the spicules experience torsional motions. Many spicules seem to show multi-thread structuring. In the modeled type II spicule the dominant acceleration and heating mechanisms vary significantly across the spicule, i.e., perpendicular to the magnetic field. For instance, the compression caused by the Lorentz force and the dissipated magnetic energy does not occur uniformly across the spicule. In fact, these processes vary in location and time, i.e., different "field lines" experience different mechanisms, and the dominant mechanism(s) at one location also evolve with time. The ejection and the expansion of the plasma is not uniformly distributed across the coronal part of the spicule. In addition, these "field lines" also experience changes in the magnetic connectivity, so both heating and acceleration jump from line to line in space and time. Most importantly, the spicule is not a structure following a confined magnetic flux tube. In short, all of these processes do not occur only along the field lines but also perpendicular to the field lines, something that is ignored in a 1D approach.

The chromosphere in our numerical simulation is significantly different from the solar chromosphere in several aspects: it is not as dynamic as revealed by observations, and type II spicules occur much more frequently on the Sun. In fact, we only have a single event that resembles (in several aspects) type II spicules. It is thus crucial to approach that deficiency in the models, and it needs to be investigated whether ion-neutral interactions, a more realistic treatment of the small-scale flux emergence, large scale motions or better spatial resolution can produce a more dynamic chromosphere. As mentioned above, the modeled ambient and emerging magnetic field is highly simplified. This may lead to the lack of dynamics and of type II spicules. It is also important to mention that other physical processes, such as reconnection, could drive type II spicules that might produce similar observables, but this needs to be investigated. Finally, type II spicules are not occurring in the 

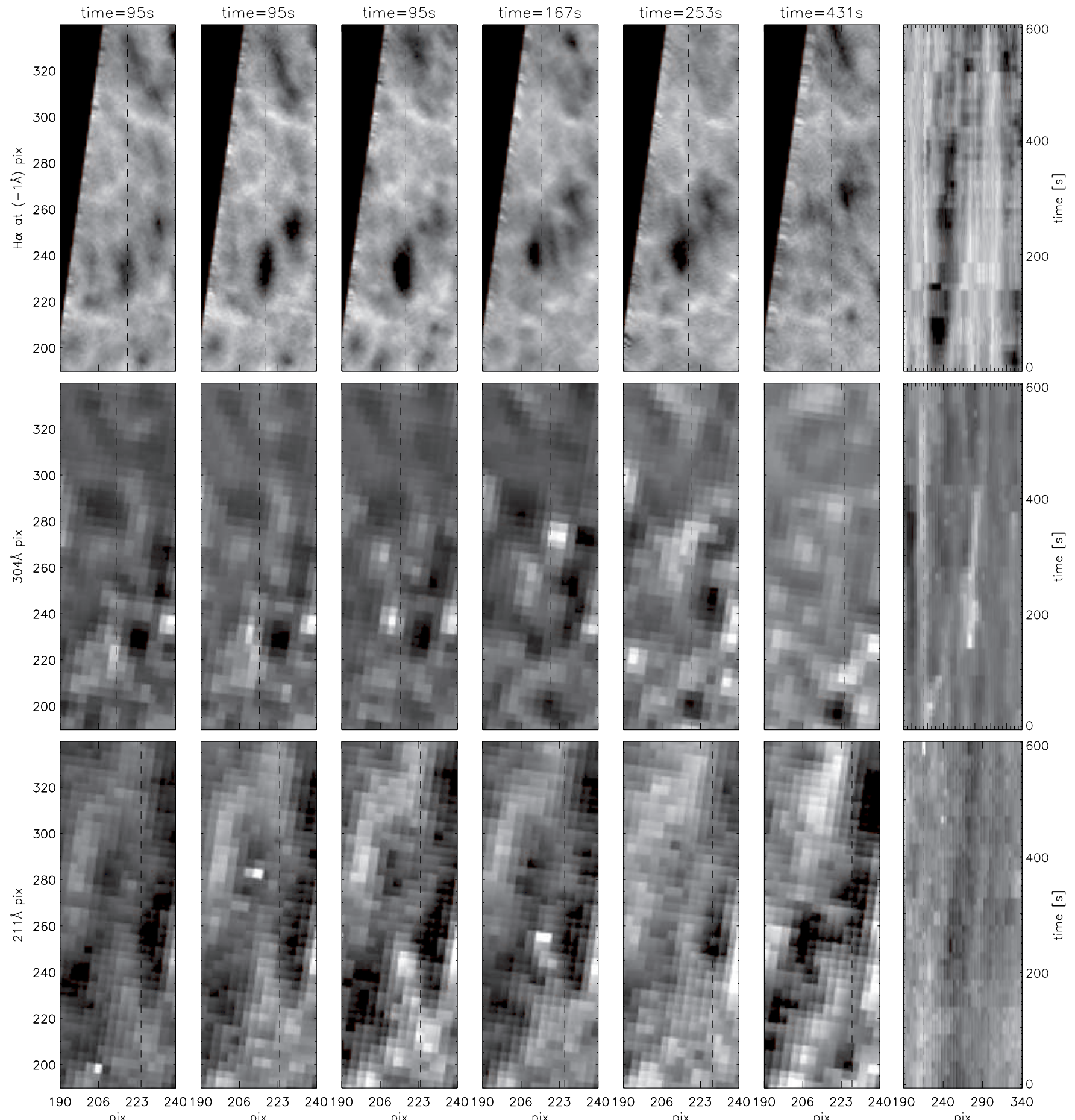

pix

Figure 20. Temporal evolution of an observed RBE visible as a dark feature in $\mathrm{H} \alpha(-868 \mathrm{~m} \AA)$ using Hinode, and associated brightenings in $304 \AA$ and $211 \AA \AA$ intensity using SDO/AIA shown from top to bottom in the left panels. Space time plots (right most column) along the axis of the RBE (dashed lines in the left panels) show a brightening in the transition region and coronal images along the spicule but also at the footpoint of the spicule. The brightening at the footpoint in $304 \AA$ and $211 \AA$ lasts for a few hundred seconds.

simulations as frequently as in the observations and the episodic nature of the RB asymmetry is missing in the models. A satisfactory model of spicules must be able to reproduce these observables too.

The research leading to these results has received funding from the European Research Council under the European Union's Seventh Framework Programme (FP7/2007-2013)/ ERC grant agreement No. 291058. We gratefully acknowledge support by NASA grants NNX08AH45G, NNX08BA99G, NNX11AN98G, NNM07AA01C (Hinode), and NNG09FA40C (IRIS). T.M.D.P.'s research was supported by the NASA Postdoctoral Program at Ames Research Center through Contract No. NNH06CC03B. H.T.'s work at CfA is supported under contract 8100002705 from Lockheed-Martin to SAO. The 3D simulation and synthesis have been run on clusters from the Notur project, and the Pleiades cluster through the computing project s1061 from the High End Computing (HEC) division 

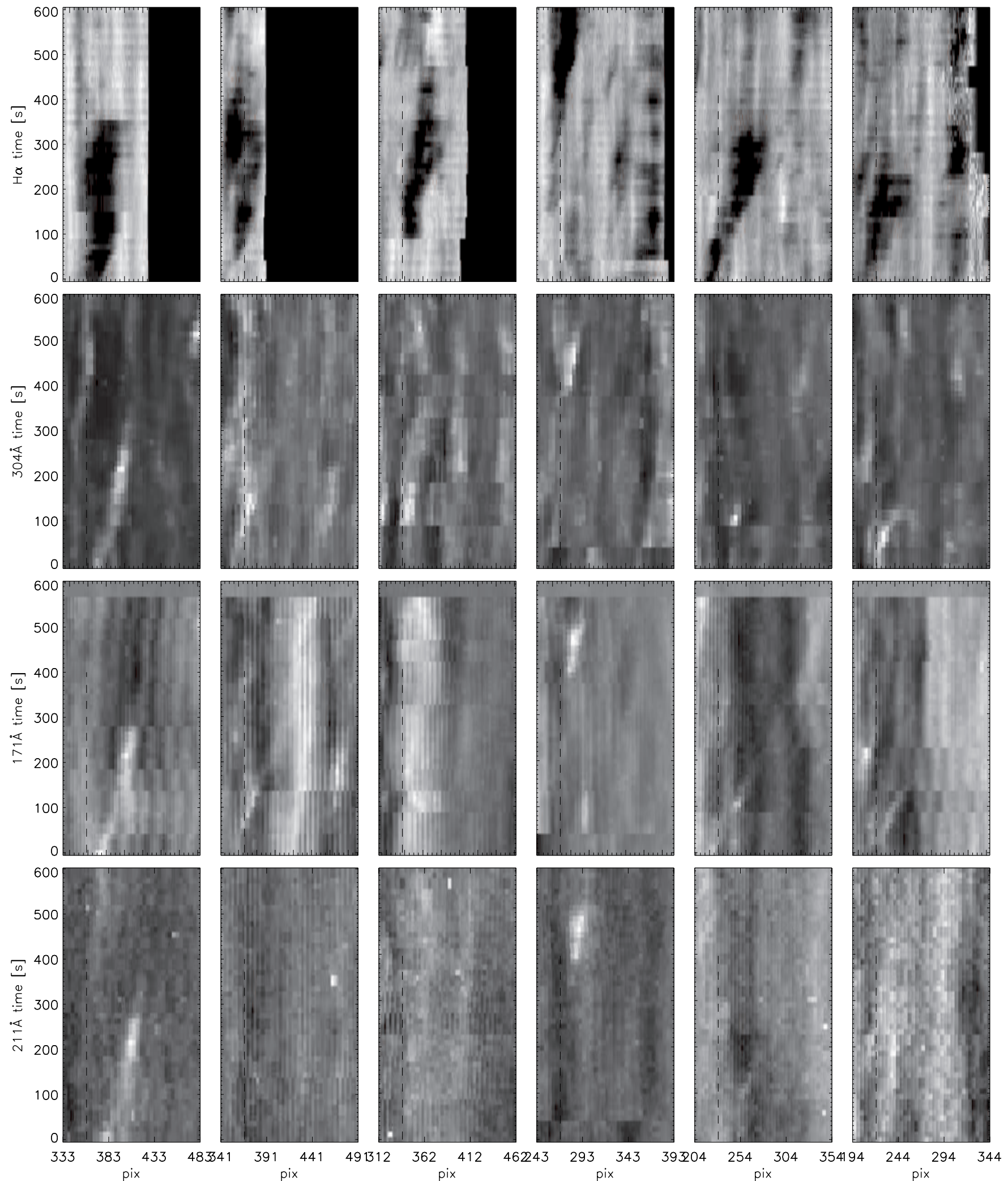

Figure 21. Space time plots along the axis of the RBE (top row) showing brightenings in transition region (second row, $304 \AA$ ) and coronal images (third and fourth row, $171 \AA$ and $211 \AA$ ) during the evolution of several spicules. In some AIA channels such brightenings also occur at the footpoints of the various spicules. The brightenings at the footpoints last for a few hundred seconds.

of NASA. We thankfully acknowledge the computer and supercomputer resources of the Research Council of Norway through grant 170935/V30 and through grants of computing time from the Programme for Supercomputing. The Swedish $1 \mathrm{~m}$ Solar
Telescope is operated by the Institute for Solar Physics of the Royal Swedish Academy of Sciences in the Spanish Observatorio del Roque de los Muchachos of the Instituto de Astrofísica de Canarias. 

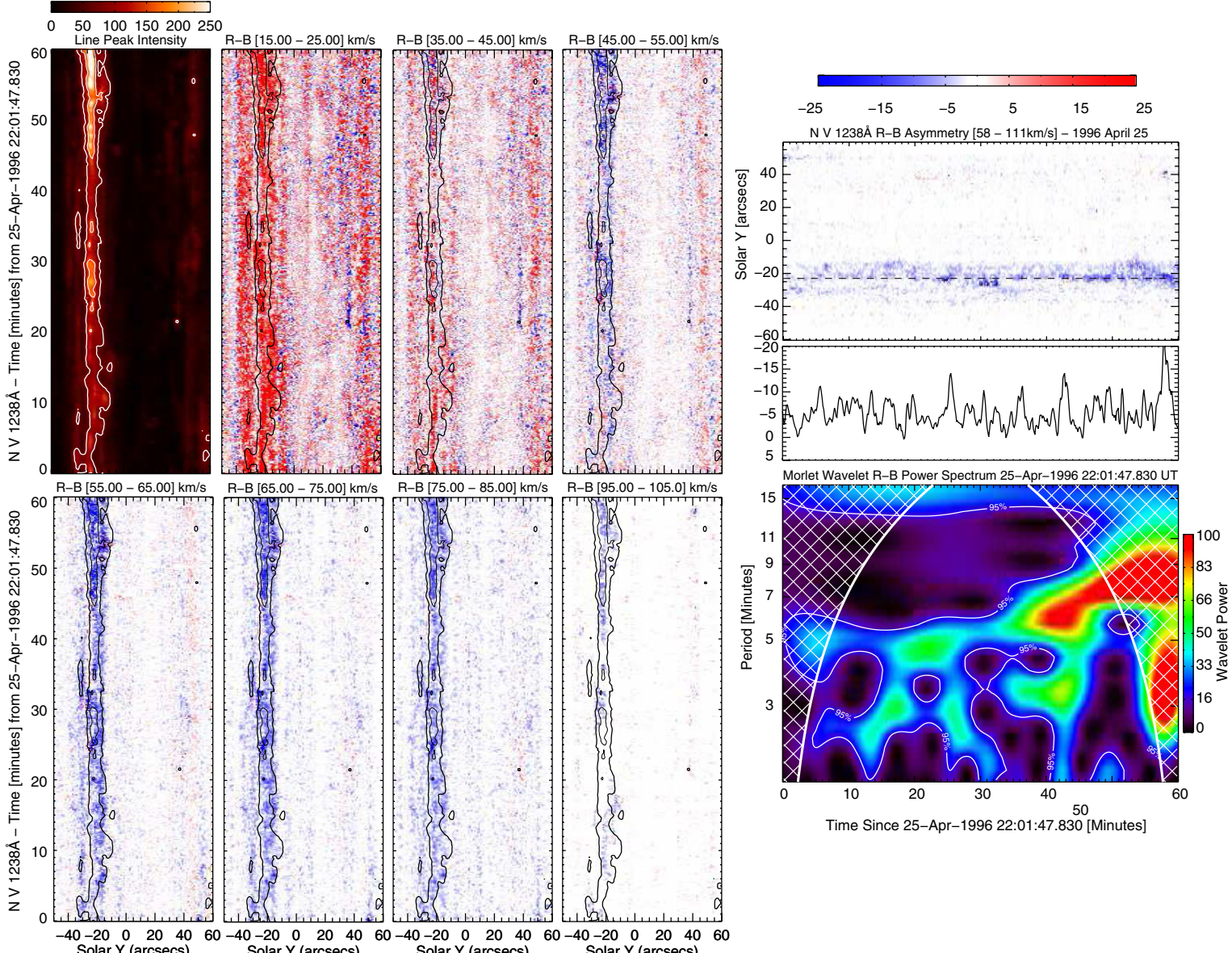

Figure 22. Time series in Nv $1238 \AA$ at disk center, in a quiet Sun region, with SUMER (left panel). Data from 1996 April 25, 22:01-23:01 UT (7.5 s exposure) SUMER quiet Sun, disk center, on the left panel rows. The panels show the peak line intensity and the R-B profile asymmetry in steps from 15 to $105 \mathrm{~km} \mathrm{~s}^{-1}$. The panels are overlaid with intensity contours at peak intensity levels of 75 and 150 counts to delineate the supergranular network boundary (thick solid lines) and the strongest network locations (thin solid lines), respectively. The right column of panels shows the wavelet analysis of the Nv $1238 \AA \mathrm{AB}^{\mathrm{N}}\left(58-111 \mathrm{~km} \mathrm{~s}^{-1}\right.$ ) time series at $y=-22^{\prime \prime}$. The bottom panel shows the cross-hatched cone of influence and contours of the $90 \%$ significance level in the time series.

(A color version of this figure is available in the online journal.)
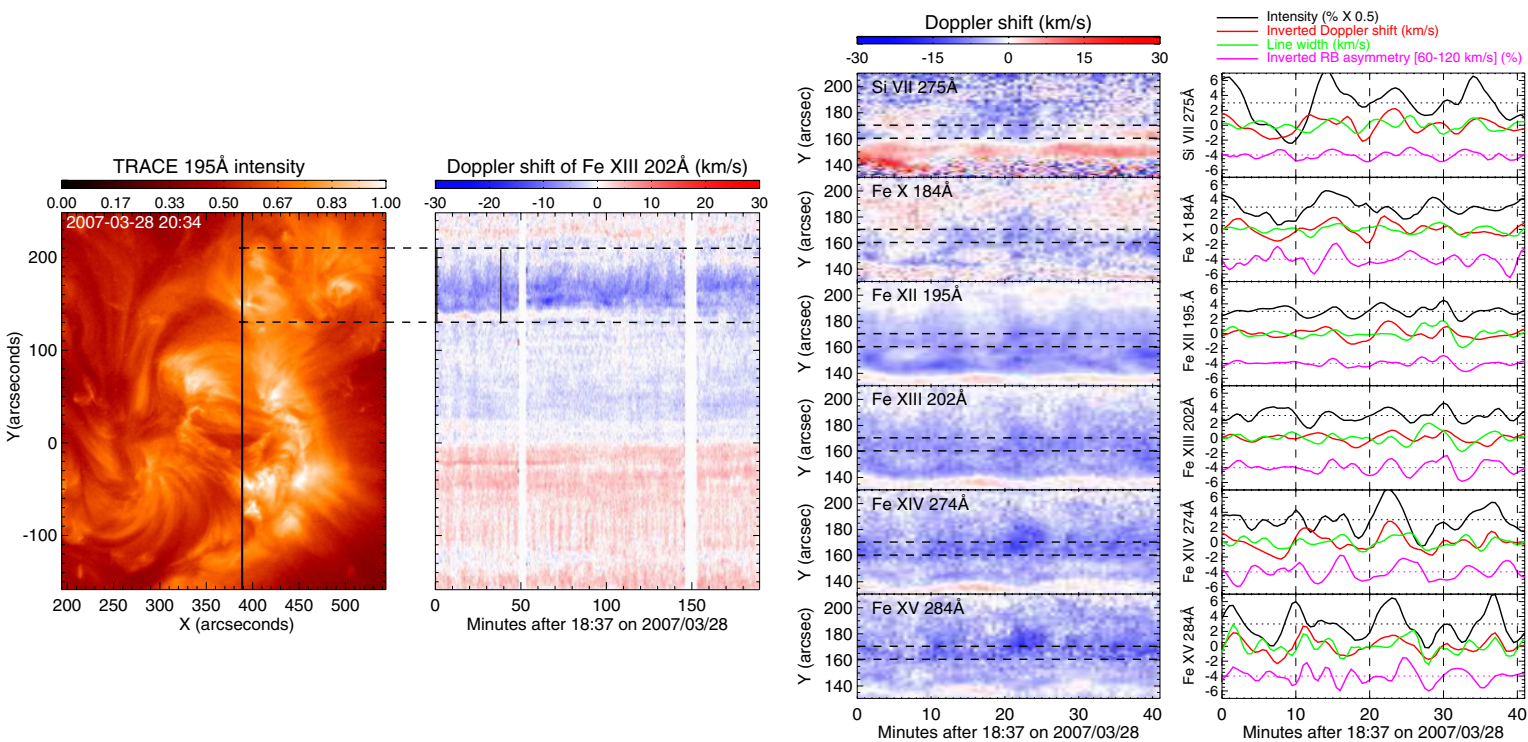

Figure 23. Adapted from Tian et al. (2012, p. 5): “Temporal evolution of the Doppler shift of several emission lines in the range of $y \sim\left[130^{\prime \prime}, 210^{\prime \prime}\right]$ in left panel. Right panel: The black, red, green, and violet curves represent respectively the line intensity, Doppler shift (inverted), line width, and RB asymmetry (inverted) averaged over the region between the two dashed lines shown in the set of panels in the third column. The intensity has been normalized to the local background and is shown as the percentage divided by 2 . The RB asymmetry has been normalized to the peak intensity of the line profile and is shown in the unit of percentage. The units of Doppler shift and line width are $\mathrm{km} \mathrm{s}^{-1}$. For the purpose of illustration, the intensity and RB asymmetry are offset by 3 and -4 respectively on the $y$-axis."

(A color version of this figure is available in the online journal.) 
To analyze the data, we have used IDL and Vapor (http://www.vapor.ucar.edu).

Eamon Scullion acquired the SST observations.

\section{REFERENCES}

Beckers, J. M. 1968, SoPh, 3, 367

Beckers, J. M. 1972, ARA\&A, 10, 73

Bradshaw, S. J. 2009, A\&A, 502, 409

Carlsson, M., \& Leenaarts, J. 2012, A\&A, 539, A39

Carlsson, M., \& Stein, R. F. 1992, ApJL, 397, L59

Carlsson, M., \& Stein, R. F. 1994, in Chromospheric Dynamics, ed. M. Carlsson (Oslo: University of Oslo), 47

Carlsson, M., \& Stein, R. F. 1997, ApJ, 481, 500

Carlsson, M., \& Stein, R. F. 2002, ApJ, 572, 626

Curdt, W., \& Tian, H. 2011, A\&A, 532, L9

De Pontieu, B., Carlsson, M., Rouppe van der Voort, L. H. M., et al. 2012, ApJL, 752, L12

De Pontieu, B., Erdélyi, R., \& James, S. P. 2004, Natur, 430, 536

De Pontieu, B., Hansteen, V. H., Rouppe van der Voort, L., van Noort, M., \& Carlsson, M. 2007a, ApJ, 655, 624

De Pontieu, B., McIntosh, S., Hansteen, V. H., et al. 2007b, PASJ, 59, 655

De Pontieu, B., McIntosh, S. W., Carlsson, M., et al. 2011, Sci, 331, 55

De Pontieu, B., McIntosh, S. W., Carlsson, M., et al. 2007c, Sci, 318, 1574

De Pontieu, B., McIntosh, S. W., Hansteen, V. H., \& Schrijver, C. J. 2009, ApJL, 701, L1

Dere, K. P. 2011, AAS Meeting, 218, 134.02

Dere, K. P., Landi, E., Young, P. R., et al. 2009, A\&A, 498, 915

Dorch, S. B. F., \& Nordlund, A. 1998, A\&A, 338, 329

Feldman, U., Ralchenko, Y., \& Doschek, G. A. 2010, ApJ, 708, 244

Georgoulis, M. K., Titov, V. S., \& Mikić, Z. 2012, ApJ, 761, 61

Grevesse, N., \& Sauval, A. J. 1998, SSRv, 85, 161

Gudiksen, B. V., Carlsson, M., Hansteen, V. H., et al. 2011, A\&A, 531, A154

Hansteen, V. H. 1993, ApJ, 402, 741

Hansteen, V. H., De Pontieu, B., Rouppe van der Voort, L., van Noort, M., \& Carlsson, M. 2006, ApJL, 647, L73

Hansteen, V. H., Hara, H., De Pontieu, B., \& Carlsson, M. 2010, ApJ, 718, 1070

Heggland, L., De Pontieu, B., \& Hansteen, V. H. 2007, ApJ, 666, 1277

Heristchi, D., \& Mouradian, Z. 1992, SoPh, 142, 21

Hyman, J., Vichnevtsky, R., \& Stepleman, R. 1979, Adv. in Comp. Meth, PDE'sIII, ed. R. Vichnevtsky \& R. Stepleman (New Brunswick: IMACS), 313

Joselyn, J. A., \& Holzer, T. E. 1975, JGR, 80, 903

Judge, P. G., de Pontieu, B., McIntosh, S. W., \& Olluri, K. 2012, ApJ, 746, 158

Judge, P. G., \& Meisner, R. W. 1994, in Solar Dynamic Phenomena and Solar Wind Consequences, the Third SOHO Workshop, ed. J. J. Hunt (ESA-SP 373; Noordwijk: ESA), 67

Klimchuk, J. A. 2012, JGRA, 117, 12102

Kosugi, T., Matsuzaki, K., Sakao, T., et al. 2007, SoPh, 243, 3

Langangen, O., De Pontieu, B., Carlsson, M., et al. 2008, ApJL, 679, L167
Leenaarts, J., \& Carlsson, M. 2009, in ASP Conf. Ser. 415, The Second Hinode Science Meeting: Beyond Discovery-Toward Understanding, ed. B. Lites et al. (San Francisco, CA: ASP), 87

Leenaarts, J., Carlsson, M., Hansteen, V., \& Gudiksen, B. V. 2011, A\&A, 530, A 124

Leenaarts, J., Carlsson, M., Hansteen, V., \& Rutten, R. J. 2007, A\&A, 473, 625

Leenaarts, J., Carlsson, M., \& Rouppe van der Voort, L. 2012, ApJ, 749, 136

Lemen, J. R., Title, A. M., Akin, D. J., et al. 2012, SoPh, 275, 17

Mackay, D. H., \& Galsgaard, K. 2001, SoPh, 198, 289

Madjarska, M. S., Vanninathan, K., \& Doyle, J. G. 2011, A\&A, 532, L1

Mariska, J. T., Doschek, G. A., Boris, J. P., et al. 1982, ApJ, 255, 783

Martínez González, M. J., Collados, M., Ruiz Cobo, B., \& Solanki, S. K. 2007, A\&A, 469, L39

Martínez-Sykora, J., De Pontieu, B., \& Hansteen, V. 2012, ApJ, 753, 161

Martínez-Sykora, J., De Pontieu, B., Hansteen, V., \& McIntosh, S. W. 2011a, ApJ, 732, 84

Martínez-Sykora, J., De Pontieu, B., Testa, P., \& Hansteen, V. 2011b, ApJ, 743,23

Martínez-Sykora, J., Hansteen, V., \& Carlsson, M. 2008, ApJ, 679, 871

Martínez-Sykora, J., Hansteen, V., \& Carlsson, M. 2009, ApJ, 702, 129

Martínez-Sykora, J., Hansteen, V., DePontieu, B., \& Carlsson, M. 2009, ApJ, 701, 1569

Martínez-Sykora, J., Hansteen, V., \& Moreno-Insertis, F. 2011, ApJ, 736, 9

Matsumoto, T., \& Shibata, K. 2010, ApJ, 710, 1857

McIntosh, S. W., de Pontieu, B., Carlsson, M., et al. 2011, Natur, 475, 477

Nordlund, A. 1982, A\&A, 107, 1

Okamoto, T. J., \& De Pontieu, B. 2011, ApJL, 736, L24

Olluri, K., Hansteen, V., \& Carlsson, M. 2013, AJ, 145, 72

Pereira, T. M. D., De Pontieu, B., \& Carlsson, M. 2012, ApJ, 759, 18

Peter, H., Gudiksen, B. V., \& Nordlund, Å. 2006, ApJ, 638, 1086

Rouppe van der Voort, L., Leenaarts, J., de Pontieu, B., Carlsson, M., \& Vissers, G. 2009, ApJ, 705, 272

Scharmer, G. B., Bjelksjo, K., Korhonen, T. K., Lindberg, B., \& Petterson, B. 2003, Proc. SPIE, 4853, 341

Scharmer, G. B., Narayan, G., Hillberg, T., et al. 2008, ApJL, 689, L69

Sekse, D. H., Rouppe van der Voort, L., \& De Pontieu, B. 2012, ApJ, 752, 108

Shelyag, S., Keys, P., Mathioudakis, M., \& Keenan, F. P. 2011, A\&A, 526, A5

Shibata, K., Nishikawa, T., Kitai, R., \& Suematsu, Y. 1982, SoPh, 77, 121

Shibata, K., \& Suematsu, Y. 1982, SoPh, 78, 333

Skartlien, R. 2000, ApJ, 536, 465

Steiner, O., \& Rezaei, R. 2012, arXiv:1202.4040

Sterling, A. C., Harra, L. K., \& Moore, R. L. 2010, ApJ, 722, 1644

Suematsu, Y., Ichimoto, K., Katsukawa, Y., et al. 2008, in ASP Conf. Ser. 397, First Results From Hinode, ed. S. A. Matthews, J. M. Davis, \& L. K. Harra (San Francisco, CA: ASP), 27

Suematsu, Y., Wang, H., \& Zirin, H. 1995, ApJ, 450, 411

Tian, H., McIntosh, S. W., Wang, T., et al. 2012, ApJ, 759, 144

Uitenbroek, H. 2001, ApJ, 557, 389

van Noort, M., Rouppe van der Voort, L., \& Löfdahl, M. G. 2005, SoPh, 228, 191

Wedemeyer-Böhm, S., \& Carlsson, M. 2011, A\&A, 528, A1 